\title{
Simultaneous Execution of Multiple Radar Tasks using OFDMA
}

\author{
by
}

Sepehr Hadizadehmoghaddam

A thesis submitted in conformity with the requirements for the degree of Master of Applied Science Graduate Department of Electrical and Computer Engineering University of Toronto

(C) Copyright 2017 by Sepehr Hadizadehmoghaddam 


\begin{abstract}
Simultaneous Execution of Multiple Radar Tasks using OFDMA

Sepehr Hadizadehmoghaddam

Master of Applied Science

Graduate Department of Electrical and Computer Engineering

University of Toronto
\end{abstract}

2017

We develop the notion of orthogonal frequency division multiple access (OFDMA) to enable a radar to execute multiple tasks simultaneously. OFDMA, like the betterknown orthogonal frequency division multiplexing (OFDM) radar, encodes information on subcarriers in the frequency domain. However, in an OFDMA-based radar, the information can correspond to independent tasks to be executed simultaneously; of specific interest here, as proof of concept, is to partition the OFDMA subcarriers to detect two targets simultaneously. Designing a broadband OFDMA signal is an efficient and resourceful way to achieve this, since, as we propose, the frequency domain information for each subcarrier can correspond to each task. Applied to a high-frequency surface wave radar, in presence of a realistic ocean clutter, our results show that the OFDMA framework allows us to combine adaptive transmit beampatterns with task execution. We also develop a subcarrier allocation scheme to maximize the probability of detection. 


\section{Acknowledgements}

I would like to sincerely thank Professor Raviraj Adve, for his cordial mentoring and guidance throughout the process. I feel immensely honored to have had a thesis supervisor who took an active interest in this project and was undoubtedly open to my ideas.

I would also like to wholeheartedly thank my family for their enduring love, support and encouragement.

This research was supported by Raytheon Canada, the Natural Sciences and Engineering Research Council (NSERC) of Canada and Defence Research and Development Canada. 


\section{Contents}

List of Tables $\quad$ vi

List of Figures $\quad$ vii

1 Introduction $\quad 1$

1.1 Literature Review . . . . . . . . . . . . . . . . . . . . 2

1.2 Motivation for OFDMA Radar . . . . . . . . . . . . . . . . . . 3

1.3 Overview . . . . . . . . . . . . . . . . . . . . 3

2 OFDMA Radar Model $\quad 5$

2.1 Signal Transmission Model . . . . . . . . . . . . . . . . 5

2.2 Signal Reception Model . . . . . . . . . . . . . . . . . . . 8

2.3 OFDMA Radar: Practical Adjustments . . . . . . . . . . . . . . 9

2.3.1 The Cyclic Prefix . . . . . . . . . . . . . . . . 9

2.3.2 High Peak-to-Average Power Ratio . . . . . . . . . . . . . . . . 11

2.3.3 Band Restricted Transmission . . . . . . . . . . . . . . . . 15

2.3.4 Task Data Leakage . . . . . . . . . . . . . . . . . . . 17

2.4 Summary . . . . . . . . . . . . . . . . . . 20

3 HFSWR Case Study $\quad 22$

3.1 Ocean Clutter . . . . . . . . . . . . . . . . . . . . 22

3.1.1 Compound K-distribution for Clutter Amplitude . . . . . . . . . . 23

3.2 OFDMA HFSWR Simulation . . . . . . . . . . . . . . . . . 27

3.2.1 Tasks Definition . . . . . . . . . . . . . . . . 27

3.2.2 OFDMA Configuration . . . . . . . . . . . . . . . . 28

3.2 .3 Symbol Assignment . . . . . . . . . . . . . . . . . . . 28

3.2 .4 PAPR Control . . . . . . . . . . . . . . . 31

3.2.5 Target Resolution of OFDMA Signals . . . . . . . . . . . . . . . . 32

3.2.6 Ocean Clutter Rejection . . . . . . . . . . . . . . . 34 
3.3 Summary . . . . . . . . . . . . . . . . . . . . 38

4 Conclusions $\quad 39$

4.1 Review .............................. 39

4.2 Contributions and Discussions . . . . . . . . . . . . . . . 40

4.3 Future Work . . . . . . . . . . . . . . . . . . . . 41

A Empirical Model for Clutter Scale Factor 43

B The Hybrid Method to Simulate Ocean Clutter 45

B.1 Shape factor to be an integer . . . . . . . . . . . . . . 46

B.2 Shape factor to be an integer plus $0.5 \ldots \ldots$. . . . . . . . . 46

B.3 Shape factor to be an arbitrary number . . . . . . . . . . . . . . 47

B.3.1 Correlation check to pass . . . . . . . . . . . . . . . 47

B.3.2 Correlation check to fail . . . . . . . . . . . . . . . 47

$\begin{array}{ll}\text { Bibliography } & 49\end{array}$

$\begin{array}{ll}\text { Bibliography } & 49\end{array}$ 


\section{List of Tables}

2.1 Parameters for an instance of OFDM radar with CP . . . . . . . . 11

2.2 Parameters for an OFDMA radar without CP, to track two targets . . . 19

3.1 HFSWR system basic characteristics for the case study . . . . . . . . . 29

3.2 Final OFDMA radar configuration . . . . . . . . . . . . 31

3.3 Implementation comparison of different PAPR reduction techniques . . . 32

3.4 Clutter rejection effect on sidelobes . . . . . . . . . . . . 37

A.1 Radar attributes used to predict clutter scale factor . . . . . . . . . . 43 


\section{List of Figures}

2.1 Cyclic Prefix mechanism . . . . . . . . . . . . . . . . . . . 10

2.2 Ambiguity for an instance of OFDM radar . . . . . . . . . . . . . 11

2.3 Clipping Effect . . . . . . . . . . . . . . . . . . . . . . . . 13

2.4 SLM block diagram . . . . . . . . . . . . . . . . . . . . . . . . . 14

2.5 Resampling Mechanisms . . . . . . . . . . . . . . . . . . 16

2.6 A realizable notched signal . . . . . . . . . . . . . . . . 17

2.7 Tracking task of two targets, with different levels of performance accuracy 19

3.1 PDF of Compound K-distribution, for varying values of $\nu \ldots \ldots 25$

3.2 Using the Hybrid Method to generate sea clutter data . . . . . . . . . 26

3.3 Effect of extensive bandwidth on OFDMA radar detection . . . . . . . 30

3.4 Task performance comparison of different PAPR reduction techniques . . 33

3.5 Number of subcarriers effect on radar task performance . . . . . . . . . 33

3.6 Benefits of OFDMA for closely juxtaposed targets tracking . . . . . . 35

3.7 Clutter shape factor effect on OFDMA radar . . . . . . . . . . . 35

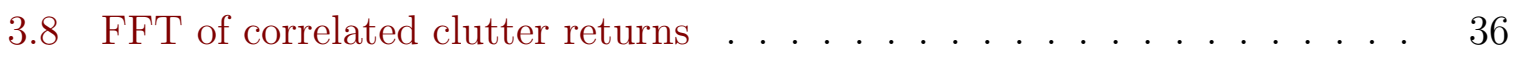

3.9 FFT of angle specific clutter returns $\ldots \ldots \ldots \ldots \ldots$

3.10 Clutter Rejection Conjecture Test . . . . . . . . . . . . . . . . . 38 


\section{Chapter 1}

\section{Introduction}

It can be argued that improvements provided by novel signal processing algorithms and techniques in recent years, are in similar scale to Moore's Law hardware advances. In the era of information fluidity and sharing, where numerous services compete for the biggest share of users, fast and adaptive data transfer schemes become extremely valuable. To this end, research efforts on the topic of radar signal processing have seen a significant resurgence, unlike the slow-paced movements in years prior.

The fundamental problem in radar is to design a waveform that upon transmission, accurately and efficiently executes the radar tasks. Any radar system must be capable of running multiple tasks such as tracking targets and surveillance; a traditional radar system executes these tasks sequentially, wherein, in a single time slot all radar resources are devoted to a single task. Therefore, radar systems can be very demanding of bandwidth. However, the advent of all-digital waveform generation allows for the real-time design of transmit waveforms; and this thesis exploits such flexibility to consider the execution of multiple tasks simultaneously. The proposed approach is based on the notion of Orthogonal Frequency-Division Multiple Access (OFDMA) applied to radar is explained, a concept borrowed from simultaneous multi-user wireless communications. Orthogonal FrequencyDivision Multiplexing (OFDM) has proven to be an extremely reliable communications technique over noisy channels with dispersion, and/or multipath fading, and thus OFDM has been adopted as the main method in many contemporary broadband communication applications, such as LTE [1]. An OFDM signal is constructed of a set of subcarriers which are orthogonal in time domain, that is, each subcarrier has an integer number of cycles over a symbol period. The adverse channel conditions are often battled with redundant block transmission of data, such as inserting cyclic prefix as time-domain guard intervals. OFDMA is the multi-user version of OFDM. Both OFDM- and OFDMA-based wireless communications systems place data on several, narrowband, subcarriers, which 
taken together form a broadband transmit signal. The key difference is that in OFDM, the data is all for one user, while in OFDMA, each user is assigned a subset of subcarriers, thereby allowing for multiuser transmissions. Importantly, in both systems, the overall signal is efficiently created using an inverse Fast Fourier transform followed by a single modulation step.

The core idea of this thesis is to propose an OFDMA framework for radar signals. To this end, it is imperative to explore the current state of OFDM radars.

\subsection{Literature Review}

The early works on OFDM radar, and associated multi-frequency radar applications, were mainly conducted by Levanon [2]. The benefits of frequency diversity in a radar signal, has been well-established in various applications; such as synthetic aperture radar [3], target detection and tracking in multipath scenarios [4], and direction of arrival estimation [5]. Based on these efforts, we can conclude that the target responses strongly depend on the frequency content of the transmitted signal [6]. However, using OFDM techniques in radar signal processing is still widely a novelty, and minimal to no implementations of such system exists in the industry. Currently, the most popular approach among researchers to an OFDM-based radar, is a system potentially capable of of simultaneously communicating wirelessly and using the wireless signal for radar tasks $[7,8]$. Since the front-end architecture of communication systems has transitioned into a digital domain, the hardware requirements for radar and wireless appear to be similar. However, these systems are usually far apart in the spectrum, due to their respective operational frequency. Using an OFDM signal, combined with Phase-Shift Keying (PSK), provides the feasibility of implementing a joint radar and communication system [9].

Alternatively, scientific contributions today have validated that a standalone radar system leveraging OFDM, features spectral flexibility and efficiency, and well-behaved ambiguity function [10]. Other notable benefits include superior range profiles [11], low interference in specific target detection scenarios [12] and a cost and space efficient alternative to autonomous landing guidance (ALG) systems [13]. In [14] it is shown that for the task of detection, OFDM and Chirp sequence radar signals, give an identical performance.

Overall it appears that salient investigations in OFDM radar, practically, have overlooked a comprehensive integration of existing, well-developed OFDM frameworks into existing, traditional radars. That is, building a signalling scheme for the radar based on OFDM concepts, with capabilities akin to commercial wireless systems such as LTE. 


\subsection{Motivation for OFDMA Radar}

It is scientifically supported that using OFDM signals in a radar system is a highly advantageous manner to radar waveform design, specially from the perspective of commercial communications. One can suggest that, the fast and efficient implementations of OFDM frameworks in contemporary wireless systems, can be brought to modern radar systems as well. Moreover, as the currently available bandwidths, within regulated limits, get more populated, a prominent demand for smart capacity allocation algorithms emerges. Along with the idea of co-habiting and co-existing wireless and radar systems, it is essential to develop all bandwidth consuming systems in the most adaptive and flexible nature possible. Such an approach may enable purposeful allocation and seamless transition between presumed functions of the system in other words, in the case of a radar, the occupied portions of its available bandwidth must be deliberately assigned to the several tasks the radar is designated for.

Hence, decomposing possible functions of a system and required frequency (or bandwidth sub-portion) assignment for each function, becomes of peak interest. This is identical to how OFDMA communication systems run as of now, where users connected to the system are allocated their own bandwidth subsets designed purposefully to the users need (e.g. texts, phone calls, video, etc.). Most importantly, all users are served simultaneously, to an acceptable standard of quality. Consequently, the motivation for this thesis, is a novel interpretation of radar tasks as virtual users, and subsequent designated communication resource allocation for each user. In other words, the OFDMA radar developed in this thesis, aims to execute tasks such as target tracking and detection, simultaneously, in a spatially efficient and flexible manner. Beyond this, we hope with the rudimentary layout of OFDMA radar, relevant ideas can be borrowed from wireless systems using OFDMA, for more sophisticated aspects of a radar system such as task scheduling, optimized power allocation, and superior robustness to dispersive propagation environment.

\subsection{Overview}

In this thesis, we design an OFDMA radar capable of executing multiple tasks simultaneously. Since advantages of such a system, are best displayed after the implementation phase; our design is focused predominantly on a practical angle. Thus, a comprehensive proof of concept is documented in the context of High Frequency Surface Wave Radar (HFSWR) systems. So, concerns such as bounded power consumption, strictly in-band 
transmission, and ocean clutter disturbance, must all be heeded to. The thesis structure is as follows: first, the basic transmission and reception signal models are explained, including chosen test statistics, and adaptive pre-processing techniques to conform to realistic scenarios. Then, we begin our case study of an OFDMA surface wave radar, by introducing the system specifications of a currently deployed HFSWR by Raytheon. Finally, in the conclusion, we summarize the topics covered in the thesis, as well as potential extensions for future work. 


\section{Chapter 2}

\section{OFDMA Radar Model}

In this chapter, we propose a model for our OFDMA radar system. The core idea is to decompose radar tasks, and treat them like (virtual) users, whose requests are served by the OFDMA signal. To this end, we integrate the OFDM signaling into a HFSWR system, with multiple transmitter and receiver antennas.

\subsection{Signal Transmission Model}

OFDM is a spread-spectrum transmission technique, in which the signal is constructed of digital data symbols on several carriers; where those carriers are orthogonal. Orthogonality is obtained by having a uniform frequency spacing in between carriers. The signal duration, is the inverse of the carrier spacing. Hence, it is trivial to design an OFDM signal given the following parameters: the digital symbols, number of subcarriers, and the overall bandwidth (or, equivalently, the duration of the signal). It must be noted that this thesis does not give the complete treatment of the OFDM signal as used in the communications; since the primary aim of ours is to establish a basic familiarity with the fundamentals of OFDM for prospective readers. Therefore, OFDM principles such as frequency synchronization, are omitted.

Let us define the number of subcarriers for an OFDM signal as $K$. So, for an available bandwidth of $B_{0}$ :

$$
\begin{aligned}
& \Delta f=\frac{B_{0}}{K} \\
& T_{\mathrm{o}}=\frac{1}{\Delta f}
\end{aligned}
$$

where $T_{\mathrm{o}}$ is the signal duration. Next, we denote the data symbols on one subcarrier 
as $I_{\mathrm{k}} \in \mathcal{C}, \quad \forall k \in\{0,1, \ldots, K-1\}$. Now we have all the basic elements to construct our OFDM time-domain signal. As per OFDM theory, once the symbols $I_{\mathrm{k}} \forall k \in$ $\{0,1, \ldots, K-1\}$ are defined, the signal is conveniently obtained by an Inverse Fast Fourier Transform (IFFT)[1]. If we let $\mathcal{F}(\cdot)$ denote the FFT operation, transmission is given as:

$$
x[n]=\mathcal{F}^{-1}\left(\left\{I_{0}, I_{1}, \ldots, I_{K-1}\right\}\right)
$$

For common implementations of the IFFT operation, it is best to choose powers of 2 for value of $K$, so that the computation becomes efficient. Finally, through pulse shaping, a continuous-time signal is achieved:

$$
s(t)=\sum_{n=0}^{K-1} x[n] p\left(t-n T_{s}\right) e^{j 2 \pi f_{c} t}
$$

in which, $f_{c}$ is the center frequency, $p(\cdot)$ is a template pulse with unit energy $(\|p(\cdot)\|=1)$, and it lasts on the order of $T_{s}$, the effective sampling period in $x[n]$ :

$$
T_{s}=\frac{T_{\mathrm{o}}}{K}=\frac{1}{B_{0}}
$$

The transmitter comprises a linear array of $N_{\mathrm{T}}$ equi-spaced elements are $d$ meters apart. Each transmit antenna is capable of transmitting its own broadband waveform comprising $K$ subcarriers, in other words, each OFDMA transmission block comprises $K$ time-domain samples created using an IFFT of $K$ frequency domain (data) symbols, placed on the $K$ subcarriers. Additionally, in an OFDMA system, each user is assigned a subset of the subcarriers. Since the objective is to design a multitasking radar system, when constructing the transmit waveform of each antenna, we use the spatial signature required by each task in the design of each of the $K$ symbols. In other words, given $Q$ tasks, we define $K(q)$ to be the set of subcarrier indexes, assigned (or allocated) to task $q, \forall q \in\{1,2, \ldots, Q\}$.

$$
\begin{aligned}
I_{n_{t}}(k, q) & = \begin{cases}I_{n_{t}, k} & k \in K(q) \\
0 & \text { otherwise }\end{cases} & \forall k \in\{0,1, \ldots, K-1\} \\
x_{n_{t}}^{(q)}[n] & =\mathcal{F}^{-1}\left(I_{n_{t}}(k, q)\right) & \forall n \in\{0,1, \ldots, K-1\}
\end{aligned}
$$




$$
x_{n_{t}}[n]=\sum_{q=1}^{Q} x_{n_{t}}^{(q)}[n]
$$

With this notation, we are now able to partition our transmission symbols. In Eq. (2.6), $I_{n_{t}, k}$ is designed particularly for each task. That is, for each task, two decisions must be made: the subcarrier subset $(K(q))$ allocated to this task, and the data symbols placed on $K(q)$ which are designated particularly for the task at hand. Later, this procedure is explained in greater detail.

By a simple extension, we can now proceed with task specific pulse shaped signals, based on Eq. (2.7) and Eq. (2.4):

$$
\begin{aligned}
s_{n_{t}}^{(q)}(t) & =\sum_{n=0}^{K-1} x_{n_{t}}^{(q)}[n] p\left(t-n T_{s}\right) e^{j 2 \pi f_{c} t} \\
s_{n_{t}}(t) & =\sum_{q=1}^{Q} s_{n_{t}}^{(q)}(t)
\end{aligned}
$$

and now we have a comprehensive, top-to-bottom approach to design waveforms on all of the radar transmitters. In order to design the complex data symbols, and the task frequency allocation regime, the knowledge of tasks at hand is needed. However, this necessity is a minimal hurdle that extends beyond the scope of this thesis, where the primary objective is to propose a fundamental layout of an OFDMA-enabled radar.

Finally, the signal that our radar transmits, is beamformed to a look angle, $\theta$. Defining the spatial frequency, $\psi(\theta)$, for the $N_{\mathrm{T}}$ antennas that are $d$ meters apart, the angle dependent transmission is given by Eq. (2.12):

$$
\begin{aligned}
\psi(\theta) & \triangleq \frac{2 \pi d}{\lambda} \sin (\theta) \\
s(t, \theta) & =\sum_{n_{t}=0}^{N_{\mathrm{T}}-1} s_{n_{t}}(t) e^{j n_{t} \psi(\theta)}
\end{aligned}
$$

equivalently, each task is associated with the angle-dependant transmit signal:

$$
s^{(q)}(t, \theta) \triangleq \sum_{n_{t}=0}^{N_{\mathrm{T}^{-1}}} s_{n_{t}}^{(q)}(t) e^{j n_{t} \psi(\theta)}
$$




\subsection{Signal Reception Model}

This section outlines the model of the received signal in the OFDMA radar. As mentioned earlier, we are mainly considering an ideal OFDM signal scheme, so apart from pulse shaping, and (later) cyclic prefix, other machinations of an OFDM system are ignored, such as frequency synchronization. We also assume prefect communication channels, however, we do take into account clutter and noise.

The antenna setup is similar to the transmission $-N_{R}$ receivers, each $d$ meters apart ${ }^{1}$. So, for the $n_{r}$-th receiver:

$$
y_{n_{r}}(t)=z_{n_{r}}(t)+c_{n_{r}}(t)+\eta_{n_{r}}(t) \quad \forall n_{r} \in\left\{0,1, \ldots, N_{R}-1\right\}
$$

where $c_{n_{r}}(t)$ models the effect of signal dependant clutter, and $\eta_{n_{r}}(t)$ models of other interferences and noise. All the useful information, such as target returns, are modeled as $z_{n_{r}}(t)$, and this part of the reception is the core of our post-processing to conclude the radar tasks. To clarify, assume this signal component consists of target returns, which is practical only if the task is of the type of detection or tracking. So, for $H$ targets:

$$
z_{n_{r}}(t)=\sum_{h=1}^{H} \alpha_{h} s\left(t-\tau_{h}, \theta_{h}\right) e^{j n_{r} \psi\left(\theta_{h}\right)} e^{j 2 \pi f_{D_{h}} t}
$$

in which, for target $h, \alpha_{h}$ is attenuation factor, is located at a range related to delay $\tau_{h}$ and angle $\theta_{h}$, with Doppler frequency $f_{D_{h}}$. The term $s(t, \theta)$ was previously defined in Eq. (2.12).

Our objective is to build a multi-task radar; thus, at the receiver end, some procedure must exist to extract pertinent information to each task, and assess task completion. In this thesis, a proof of this concept is outlined. Therefore, let the tasks be the tracking of all $H$ targets, that is, prior knowledge is available for position of targets, their velocity, and their count. This yields that $Q=H$. This is a one-to-one relationship, as task $q$ is only the tracking of target $h$, and nothing else. Then to extract pertinent parts of $y_{n_{r}}(t)$ to task $q$, after beamforming, a match filtering to signal associated to the task step must be in place. That is:

$$
\zeta_{n_{r}, q}(\tau)=\int y_{n_{r}}(t) \overline{s^{(q)}}(t-\tau, \theta) d t
$$

note that $\overline{(.)}$ is the conjugate operator. Eq. (2.16) expresses the filter that matches the

\footnotetext{
${ }^{1}$ For convenience, we assume same distance between transmitter and receiver antennas
} 
received signal in Eq. (2.14), to the portion of transmission related to task $q$, or Eq. (2.13). Thus the $\zeta_{n_{r}, q}(\tau)$, calculated at each receiver, includes useful data for a specific task. The last step is to beamform this data sequence, to each task's corresponding angle, $\theta_{q}$ :

$$
r^{(q)}\left(\tau, \theta_{q}\right)=\sum_{n_{r}=0}^{N_{R}-1} e^{-j n_{r} \psi\left(\theta_{q}\right)} \zeta_{n_{r}, q}(\tau)
$$

with that, our task-based MIMO radar model is complete. This signal can be used for further processing for the task, such as comparison to a threshold for detection.

It is worth mentioning that our waveform design here, follows the fundamental approach to that of conventional radar signal design. The transmission expressed in Eq. (2.12), is constructed based on a given Pulse Repetition Frequency (PRF), and pulses of this signal would be sent by the radar to execute its supposed tasks.

\subsection{OFDMA Radar: Practical Adjustments}

To this point, the fundamentals of an OFDMA radar was explained, from transmission waveform design steps, to post-reception analysis. In the following sections, we describe the possible practical problems of the proposed system, as well as beneficial extensions to the model, such as adaptive processing, and efficient power consumption. Overall, the focus is on improving the OFDMA signal generation procedure, for better task execution performance. So, the upcoming discussion points are oriented towards overlooked issues of OFDMA systems, in order to make the radar system realistic.

\subsubsection{The Cyclic Prefix}

The term Cyclic Prefix (CP), in communications, is defined as prefixing a symbol with a repetition of the end. It serves both as a guard interval, and a mathematical convenience to characterize frequency-selective channels. In communications, OFDMA systems must use a $\mathrm{CP}$ to ensure subcarriers remain orthogonal after propagation through frequency selective channels [15]. The CP refers to appending a copy of the last few OFDMA symbols to before the OFDMA block. The length of the required prefix is set by the length of the frequency selective channel.

The principle of CP method is shown in Fig. 2.1, where $T_{p}$ is the length of the prefix, a design factor in an OFDM system. By extending the signal length, the risk of data corruption in the communication is decreased. In communications, this approach removes the effect of intersymbol interference. 
This work is developing an OFDMA radar, so, the advantages of using CP in our system must be carefully examined. To justify the implementation of OFDMA radar - an outlandish idea in its infancy - all possible bottlenecks should be avoided. So, we now explore if there are any unknown disadvantages in using Cyclic Prefix technique in an OFDMA radar. To this end, we examine the ambiguity function of a basic radar system, that uses OFDM signals, with guard intervals.

The ambiguity function, $\chi(\tau, f)$, is a two-variable function of time delay and Doppler frequency, which shows the distortion of a returned pulse due to the receiver matched filter due to the Doppler shift of the return from a moving target. The radar ambiguity function is normally used by radar designers as a means of studying different waveforms. It can provide insight about how different radar waveforms may be suitable for the various radar applications. It is also used to determine the range and Doppler resolutions for a specific radar waveform. For the OFDMA radar, whose transmission signal $s_{c p}(t)$ includes the Cyclic Prefix, the ambiguity function is given by Eq. (2.18).

$$
\chi(\tau, f)=\int_{-\infty}^{\infty} s_{c p}(t) s_{c p}^{*}(t-\tau) e^{j 2 \pi f t}
$$

We observe the effects of CP signals through an example. Let $N_{\mathrm{T}}=N_{R}=1$. We choose a $170 \mathrm{kHz}$ bandwidth, with $K=16$ subcarriers. Thus the frequency distance between subcarriers is $10.625 \mathrm{KHz}$ (Eq. (2.1)), and the signal duration is $94.11 \mathrm{\mu s}$. Our OFDM sampling time is $T_{s}=5.88 \mu \mathrm{s}$, so we choose our prefix length as $T_{p}=T_{s}$, and $N_{p}=1$. Let $s_{c p}(t)$ denote the extended signal with duration $T_{p}+T_{\mathrm{o}} \approx 100 \mu \mathrm{s}$. This means the discrete time version, $x_{c p}[n]$, is a sequence of $K+N_{p}=17$ samples. For purposes of MATLAB simulation, we use $x_{c p}[n]$. Finally, since this is a basic characterization of OFDMA radar, we choose the data symbols as from a complex Gaussian distribution with zero mean and unit variance, or $I_{\mathrm{k}} \sim \mathcal{C N}(0,1) \forall k \in\{0,1, \ldots, K-1\}$.

We need to define our PRF to get a Doppler analysis. Since each OFDM block is $100 \mathrm{us}$ long, then $P R F=10 \mathrm{kHz}$. We assume a transmission of $M=256$ OFDM pulses. With these parameters (summarized in Table 2.1), our OFDM capable radar ambiguity function is depicted in Fig. 2.2. It is evident that the redundancy in $s_{c p}(t)$, is limiting the radar range extent. In other words, the range cells in the tail end, of length $T_{p}$, in the

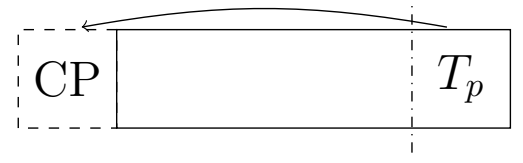

Figure 2.1: Cyclic Prefix mechanism 


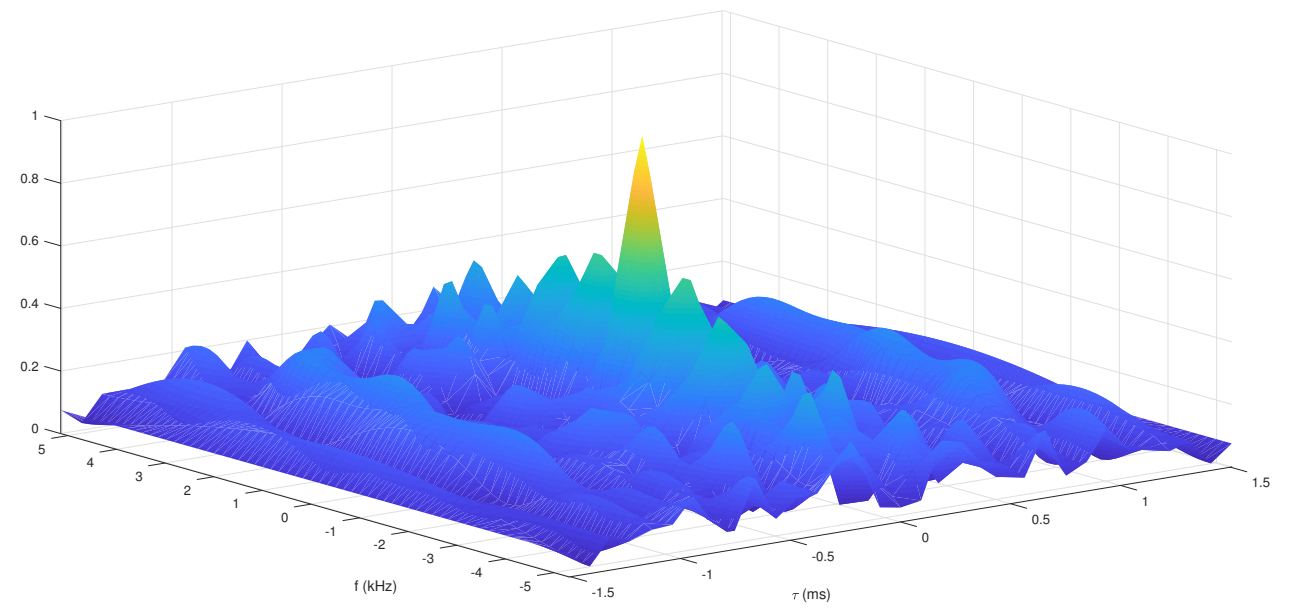

Figure 2.2: Ambiguity for an instance of OFDM radar

transmission signal $s_{c p}(t)$, will have a non-negligible product in evaluation of Eq. (2.18), $\forall \tau: m T_{\mathrm{o}} \leq \tau \leq m T_{\mathrm{o}}+T_{p}$, for an arbitrary integer value of $m \leq M$. Therefore, using a cyclic prefix, limits the radar range extent (to the equivalent delay) of $T_{s}$. However, this restriction might be practically irrelevant, specially since the value of $T_{\mathrm{o}}$ is determined by the available bandwidth and number of subcarriers, design parameters of the OFDMA radar. Particularly, in the case study of HFSWR, we can ignore deploying the CP guard.

\begin{tabular}{|c|c|}
\hline Parameter & Value \\
\hline$K$ & 16 \\
$B_{0}$ & $170 \mathrm{kHz}$ \\
$N_{\mathrm{T}}$ & 1 \\
$N_{R}$ & 1 \\
$T_{p}\left(N_{p}\right)$ & $5.88 \mu \mathrm{s}(1 \mathrm{sample})$ \\
$f_{c}$ & $3.25 \mathrm{MHz}$ \\
$\mathrm{PRF}$ & $10 \mathrm{kHz}$ \\
\hline
\end{tabular}

Table 2.1: Parameters for an instance of OFDM radar with $\mathrm{CP}$

\subsubsection{High Peak-to-Average Power Ratio}

The optimal performance of any communication system, strongly depends on the power consumption and characteristics of signals, transmitters, channels, and receivers within the system. One of the many metrics to examine in the system, is the Peak-to-Average 
Power Ratio (PAPR) of propagating signals, defined in Eq. (2.19).

$$
\begin{gathered}
\operatorname{PAPR}_{d B}(x[\cdot])=20 \log _{10} \frac{\max |x[\cdot]|}{x_{r m s}} \\
x_{r m s} \triangleq \sqrt{\frac{x[0]^{2}+x[1]^{2}+\ldots+x\left[N_{x}-1\right]^{2}}{N_{x}}} N_{x}: \text { Length of } \mathrm{x}
\end{gathered}
$$

For all complex sinusoidal waves, the PAPR is $0 \mathrm{~dB}$. In Eq. (2.3), we see the role of IFFT operation in forming an OFDM signal, and IFFT is a modulated sum of sine waves. Hence, OFDM signals have inherently high PAPR values, which are considerably above single-carrier signals. This is one of the most detrimental aspects of an OFDM scheme, since it directly increases the quantization noise of analog-to-digital converters, while the efficiency of transmitter power amplifiers is also degraded.

PAPR reduction has been an active and thriving research area in wireless communications. In an excellent survey on the topic [16], a large variety of PAPR reduction techniques are explained and compared. Generally, there are two flavors to the techniques: modifying data symbols in the frequency domain, or amplitude modulation in the time domain.

It is imperative in our application to minimize the loss of phase information in the signal (after modifications), in order to preserve angle dependency of the OFDMA data symbols. Also it is needed to keep the system computationally efficient and realizable digitally. As the results in [16] indicate, clipping (in time domain), and Selective Mapping (SLM) (in symbol domain), have the highest fitness to our requirements.

\subsubsection{Clipping Technique}

Clipping is a popular technique that bounds the magnitude of a signal, while maintaining its phase. The clipping can be two sided - where the modified signal amplitude is stapled for a value between $A_{U B}$ and $A_{L B}$.

$$
\begin{aligned}
& \tilde{x}(t)= \begin{cases}A_{U B} e^{j \angle x(t)} & |x(t)|>A_{U B} \\
x(t) & \text { otherwise }\end{cases} \\
& \tilde{\tilde{x}}(t)= \begin{cases}A_{U B} e^{j \angle x(t)} & |x(t)|>A_{U B} \\
A_{L B} e^{j \angle x(t)} & |x(t)|<A_{L B} \\
x(t) & \text { otherwise }\end{cases}
\end{aligned}
$$

By enforcing an upper bound on the magnitude of the signal, we are decreasing the 
numerator in Eq. (2.19), and thus PAPR is reduced. Unfortunately, Eq. (2.21) does not give an analytic relationship between the PAPR of an original signal and its clipped version. So, choosing values for $A_{U B}$ (and $A_{L B}$ ) requires trial and error. Obviously, $A_{U B}$ must be lower than $\max [x(\cdot)]$, and a tighter range between $A_{U B}$ and $A_{L B}$ would lower the PAPR further.

To deploy clipping in the OFDMA radar, we should mention that since our choice of the pulse shaping template, $p(\cdot)$, has a unit energy, then clipping the signal at each transmitter would lead to identical results (for fixed values of $A_{U B}$ and $A_{L B}$ ). In current literature, the clipping is applied to $x_{n_{t}}[n]$ as well; immediately after taking the IFFT of symbols [16]. Using an iterative approach, the application of clipping is mostly done at the transmitters. However, based on the required Signal-to-Noise Ratio (SNR), some noise mitigation techniques might be necessary at the receivers, to increase the spectral efficiency of the clipped signal. This method promises to preserve the phase information, so it should not deteriorate the task performance of the radar, with the designed waveform of Section 2.1. This hypothesis is tested (and proven) in the next chapter.

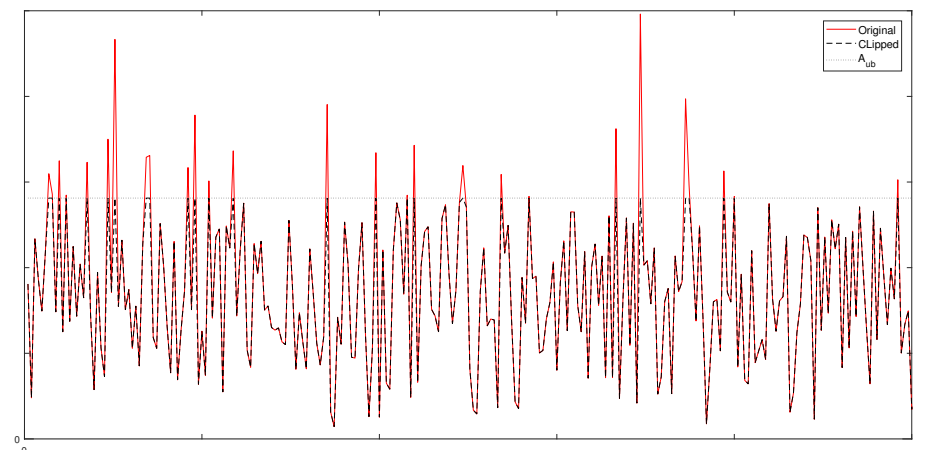

(a) Amplitude modulation

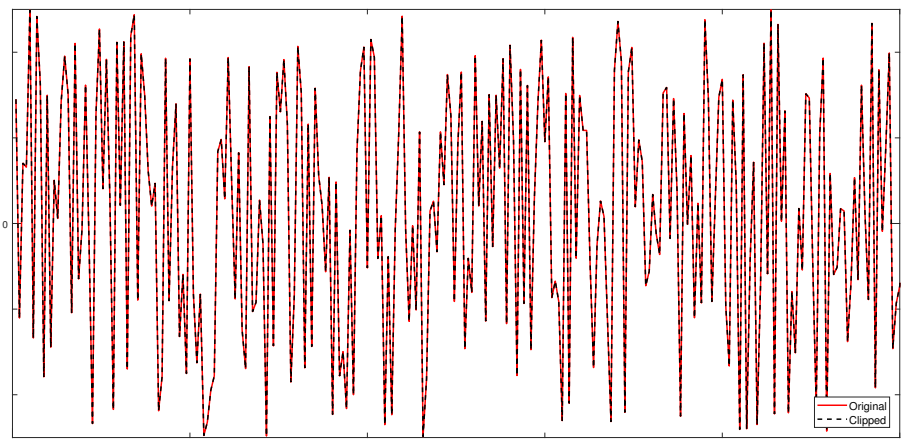

(b) Clipped and original signal have the same phase

Figure 2.3: Clipping of a random signal 


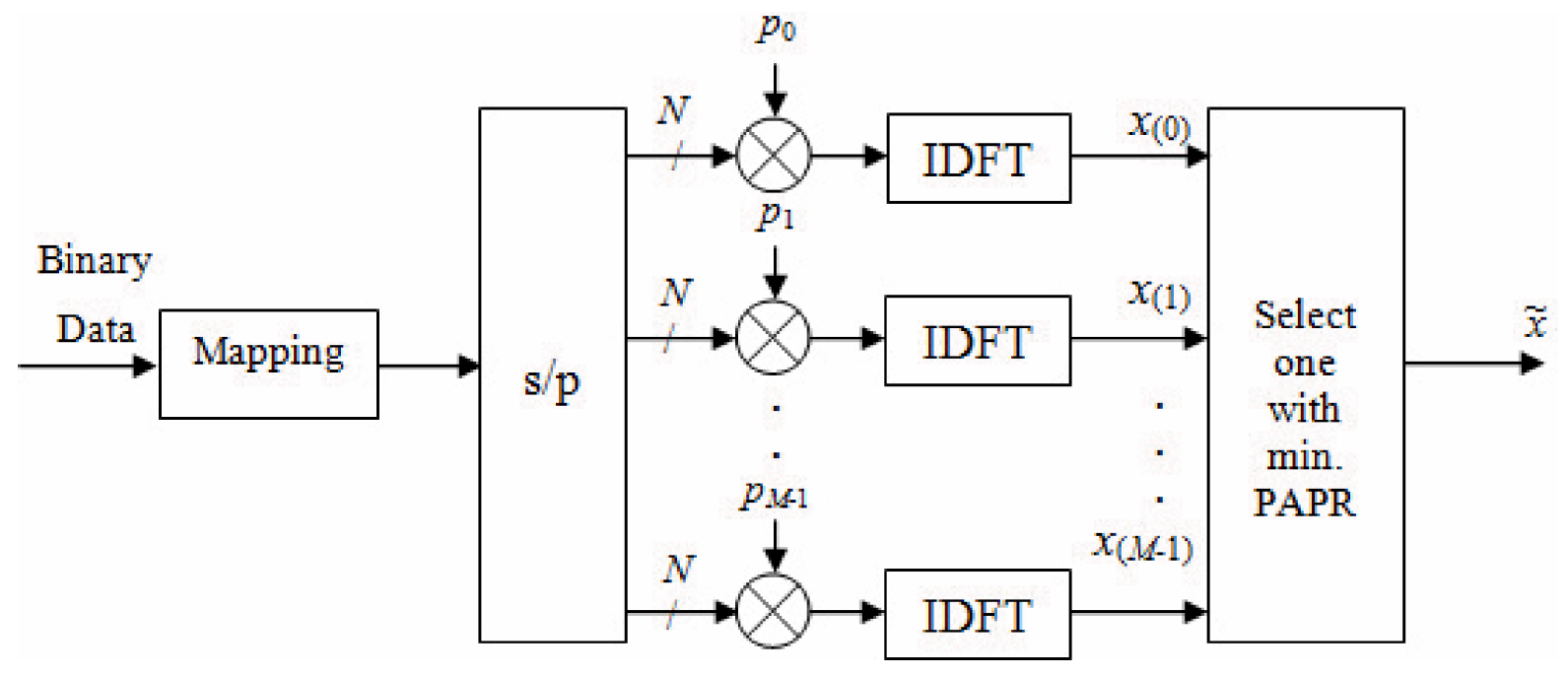

Figure 2.4: Block diagram of SLM scheme [16]

\subsubsection{Selective Mapping Technique}

Selective Mapping is the second PAPR reduction technique investigated. After a brief outline, the application to OFDMA radar is examined. Unlike clipping, SLM is an efficient, distortion less technique, without an increase in power requirement, and incurring data rate loss. The basic idea of SLM is to generate several OFDM symbols as potential candidates and then select the one with the lowest PAPR for actual transmission [16]. Traditionally, the transmission of side information is needed so that the receiver can extract the selected candidate in the transmission. The side information is minimal and the loss in data rate is usually negligible [16]. It mainly consist of the phase vector, $p_{u_{*}} \in \mathcal{C}^{1 \times K}$, where $u_{*} \in\{0,1, \ldots, U-1\}$ is the index of the generated sequence with the minimum PAPR, and $\tilde{x}=x_{\left(u_{*}\right)}$. An overview of this SLM process is found in Fig. 2.4.

Evidently, SLM is highly flexible and easy to implement. However, the choice of the distribution for phase vectors, and the number of them $(U)$, may increase the computation load. Moreover, if the desired PAPR needs a large reduction, $U$ would increase too, and based on available hardware, it might be infeasible to search for the desired data sequence. There are also concerns regarding protection of side information, as it is the key to retrieve the original symbols correctly at the receiver, but that is beyond the scope of this correspondence, and (in this regard) an ideal communication is assumed. The biggest feature of SLM, which makes it suitable to our OFDMA radar, is the flexibility of $p_{u}$ vectors, which we can utilize as an ad-hoc extension to our task-based data symbol design, the $I_{\mathrm{k}} \mathrm{s}$. We execute two separate SLM schemes in Chapter 3, and compare their performance with the performance of clipping.

That concludes the brief look into our chosen solutions to the high PAPR problem. We 
selected the two modern, easy to implement, and computationally efficient methods, that do not corrupt phase information as their mean to reduce PAPR. The direct application of both clipping and SLM to the OFDMA radar, with more defined details, requires practical constraints. All of this is discussed in Chapter 3.

\subsubsection{Band Restricted Transmission}

One of the major practical aspects of any radar system, is to keep transmission in-band, and suppress out-of-band emissions below a regulated threshold. As mentioned in Chapter 1, radar systems must aim to be as economic as possible with regards to bandwidth usage. The bands are getting increasingly occupied too, so, it is increasingly important suppress out-of-band signals, to decrease possible interference with systems of neighboring bands. The crowded bandwidth is evident in [17], which includes a comprehensive account of bandwidth allocation and regulations in United States.

Therefore, a spectrum-flexible waveform design process in every radar is very favorable. Fortunately, an OFDMA signal is renowned for this characteristic. However, in the essence of practicality, the designed waveforms need to be realizable. Much like ideal filters, a signal with sharp cutoffs at several frequencies, is physically unrealizable and difficult to approximate. So the Power Spectral Density (PSD) of transmission signal, $|X(f)|$ must decrease to zero gradually rather than abruptly. This can be achieved by basic interpolation. We are also interested in tightening the transmission to the available bandwidth $\left(B_{0}\right)$, thus, all filters applied to $X(f)$ should take this constraint into account. Moreover, in an OFDM signal, the number of frequency samples is determined by $K$, and is limited. So, if there are abrupt cutoffs in $X(f)$, for the interpolation to take effect, a higher number of frequency samples is required.

Therefore, to make physically realizable, band-restricted OFDM signals, three steps follow: 1. upsampling (by a factor of $L$ ), 2. interpolation with a band-pass filter, 3. decimation (by a factor of $L$ ). A snapshot of this system, explained in great detail in [18], can be viewed in Fig. 2.5.

There are several choices for the filter. In digital communications, problems similar to ours are resolved using a Raised-Cosine filter [19]. Considering our concern about out-of-band emissions, a more flexible filter choice is required. Generally, FIR filters are implemented using the window method; the Kaiser window, is a popular and customizable option. The cutoff frequencies, and the attenuation levels, all determine the order and length of the Kaiser window, and thus, the resulting filter [18].

We conclude this section with an example, whose parameters are defined in Table 2.1. 


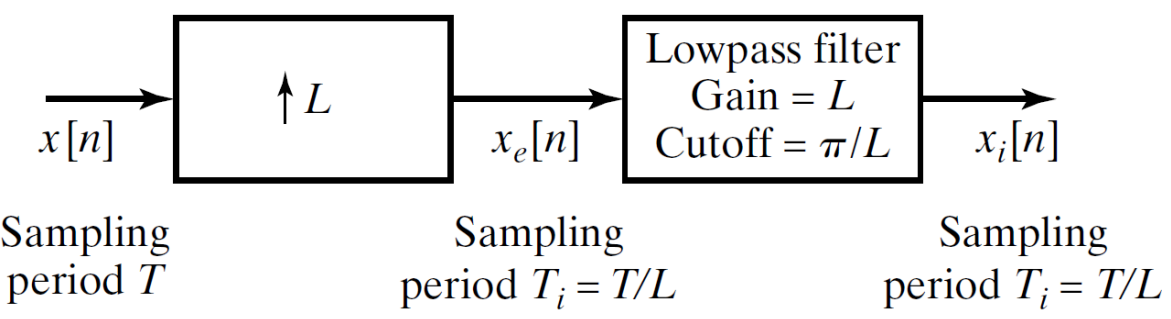

(a) upsample and interpolate

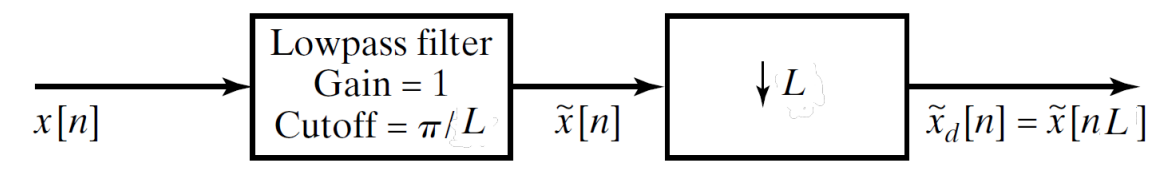

Sampling
period $T$

Sampling

Sampling

period $T$

period $T_{d}=L T$

(b) downsample and decimate

Figure 2.5: Standard mechanisms to change signal sampling rate [18]

The $I_{\mathrm{k}}$ symbols here, are chosen from 4-QAM constellation. Assume the OFDMA radar is not allowed to transmit in a $20 \mathrm{kHz}$ band of $B_{0}$, from $40 \mathrm{kHz}$ to $60 \mathrm{kHz}$. In practice, that means a signal power below some threshold in the restricted band relative to the peak. Usually a $60 \mathrm{~dB}$ drop-off is desirable. Since we are using OFDM signals, each frequency corresponds to a subcarrier, with the relationship:

$$
\begin{gathered}
f_{k}=f_{c}+k \Delta f \Rightarrow k=\frac{f_{k}-f_{c}}{\Delta f} \\
\forall k: f_{k}-f_{c} \in[40,60] \mathrm{kHz} \Rightarrow I_{\mathrm{k}}=0
\end{gathered}
$$

then band restriction can be represented in terms of data symbols, as in Eq. (2.24). Define $L=20$ as the pre-interpolation upsampling rate $^{2}$. With that, we have the ingredients to design our Kaiser filter ${ }^{3}$. Note the gain adjustments depicted in Fig. 2.5 for the filters.

Fig. 2.6 illustrates this process, where spectrum magnitude is in dB. Note that the attenuation from passband to cutoff in the low-pass filter, is the desired $60 \mathrm{~dB}$, and it is larger in out-of-band frequencies. The realized signal, is minimally affected by this out-of-band emission. The filter is designed with a 1450 long Kaiser window, and shape factor of 5.6533. It must be noted that a higher value for $K$, decreases the need for large $L$ values, which in turn simplifies the Kaiser window computation. Therefore, our proposed procedure to apply band restrictions to an OFDM signal proves effective. The process is

\footnotetext{
${ }^{2}$ Using integer values for $L$ facilitates implementation.

${ }^{3}$ We obtain the filter leveraging the MATLAB built-in function kaiser, whose parameters are calculated according to Eq.(5.72-76) in [18]
} 


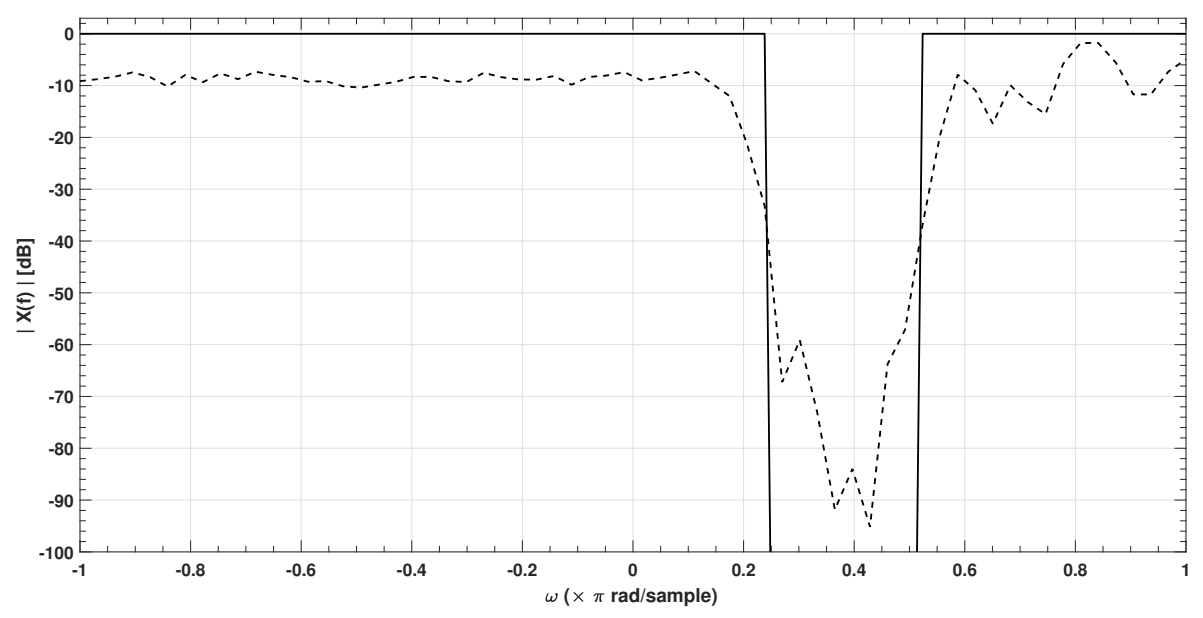

(a) Original OFDM signal (solid) and realized signal (dashed)

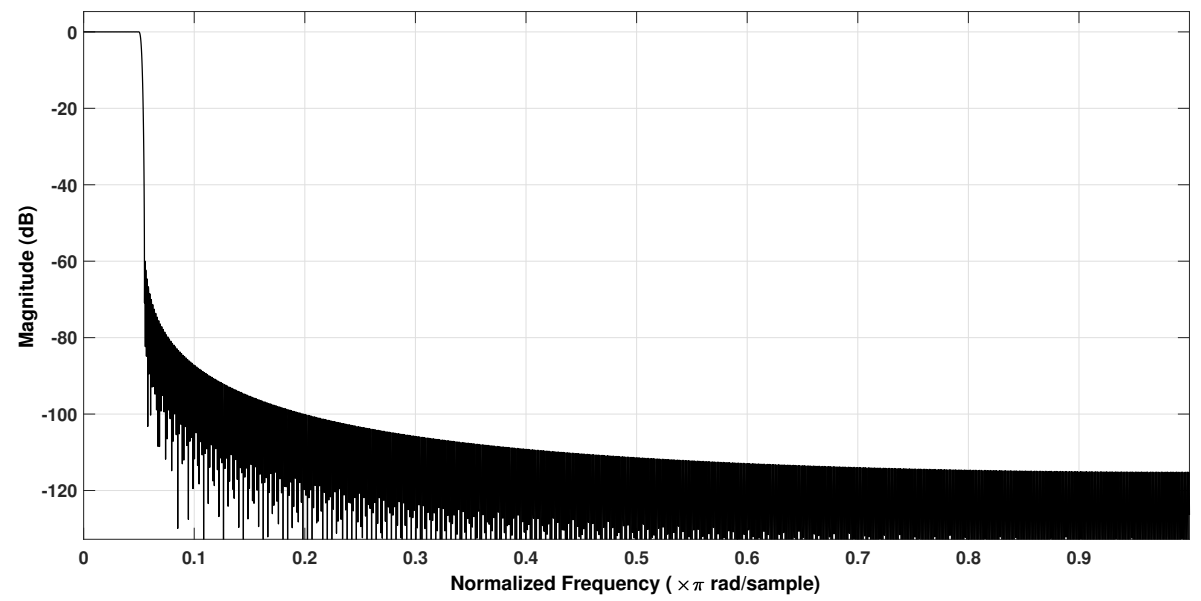

(b) The low-pass filter used in interpolation

Figure 2.6: A realizable notched signal

highly adjustable if adding several notches, with varying lengths, becomes necessary.

One practical drawback of filtering, is the changes in PAPR. Per our previous discussion, techniques are available to restrict PAPR within desired constraints. Because of our modular approach to both problems, cascading the solutions is rather straightforward.

\subsubsection{Task Data Leakage}

The practical issue of this section, is not trivial at first glance. The term 'leakage' appears worrying, as a big appeal of OFDM signals is the orthogonality, which supposedly guards against intersymbol interference. However, we are attempting to leverage OFDM in the context of radar systems, where signal returns are influenced by the physical environment. The basic target detection is possible in radars because the reflection off of a target, interactively, changes the characteristics of the arrived signal. Now, as an example, if 
our OFDMA radar is required to complete several tracking tasks simultaneously, we can expect a trace of all targets in every task return sequence (Eq. (2.17)). This phenomena is exacerbated by having multiple transmitter and receiver antennas. Therefore, to achieve accuracy, we must guarantee task returns are entirely focused on one task and one task only, immediately from the transmission. This is the core idea behind Zero-Forcing (or nullsteering). Zero-Forcing (ZF) is a method of spatial signal processing by which the multiple antenna transmitter can null multiuser interference signals in wireless communications. The underlying rule is to force the intersymbol interference to be zero, which ZF achieves through an easy and straightforward implementation.

The discussion so far has not specified any tasks at hand. As proof of concept, we focus on the detection of two targets at pre-defined angles, $\theta_{1}$ and $\theta_{2}$, with respect to the radar. Such a situation could arise in the tracking of two targets in directions $\theta_{1}$ and $\theta_{2}$.

In order to complete these tasks, we propose the following subcarrier symbol assignment: The even-indexed subcarrier symbols focus on target 1, and the odd-indexed subcarrier symbols focus on target 2 . The simplest approach is to use the beamforming weights to steer the transmission toward the two chosen angles. That is:

$$
\begin{aligned}
\boldsymbol{a}(\theta) \triangleq\left[\begin{array}{lll}
1 & e^{j \psi(\theta)} \quad \ldots & e^{j\left(N_{\mathrm{T}}-1\right) \psi(\theta)}
\end{array}\right]^{T} \\
I_{n_{t}}(k, 1)=\left\{\begin{array}{ll}
\boldsymbol{a}\left(\theta_{1}\right)_{n_{t}} & k \in K(1) \\
0 & \text { otherwise }
\end{array}: K(1)=\{0,2, \ldots, K-2\}\right. \\
I_{n_{t}}(k, 2)=\left\{\begin{array}{ll}
\boldsymbol{a}\left(\theta_{2}\right)_{n_{t}} & k \in K(2) \\
0 & \text { otherwise }
\end{array}: K(2)=\{1,3, \ldots, K-1\}\right.
\end{aligned}
$$

Eq. (2.25) defines the transmission steering vector $\left(\in \mathcal{C}^{N_{T} \times 1}\right)$, and we denote its $i^{\text {th }}$ entry with $\boldsymbol{a}(\theta)_{i}$. With this expression, our ZF goal can be achieved by redefining new steering vectors, that are the solution to the optimization problem of Eq. $(2.27) \cdot(\cdot)^{H}$ denotes the Hermitian operation.

$$
\begin{aligned}
\tilde{\boldsymbol{a}}\left(\theta_{1}\right) & =\underset{\tilde{\boldsymbol{a}}(\theta)}{\arg \max }\left|\tilde{\boldsymbol{a}}^{H}(\theta) \boldsymbol{a}\left(\theta_{1}\right)\right| \\
\text { s.t. } & \boldsymbol{a}^{H}(\theta) \boldsymbol{a}\left(\theta_{2}\right)=0 \\
\Rightarrow \tilde{\boldsymbol{a}}\left(\theta_{1}\right) & =\left(1-\frac{\boldsymbol{a}\left(\theta_{2}\right) \boldsymbol{a}^{H}\left(\theta_{2}\right)}{\left\|\boldsymbol{a}\left(\theta_{2}\right)\right\|_{2}}\right) \boldsymbol{a}\left(\theta_{1}\right) \\
\Rightarrow \tilde{\boldsymbol{a}}\left(\theta_{2}\right) & =\left(1-\frac{\boldsymbol{a}\left(\theta_{1}\right) \boldsymbol{a}^{H}\left(\theta_{1}\right)}{\left\|\boldsymbol{a}\left(\theta_{1}\right)\right\|_{2}}\right) \boldsymbol{a}\left(\theta_{2}\right)
\end{aligned}
$$




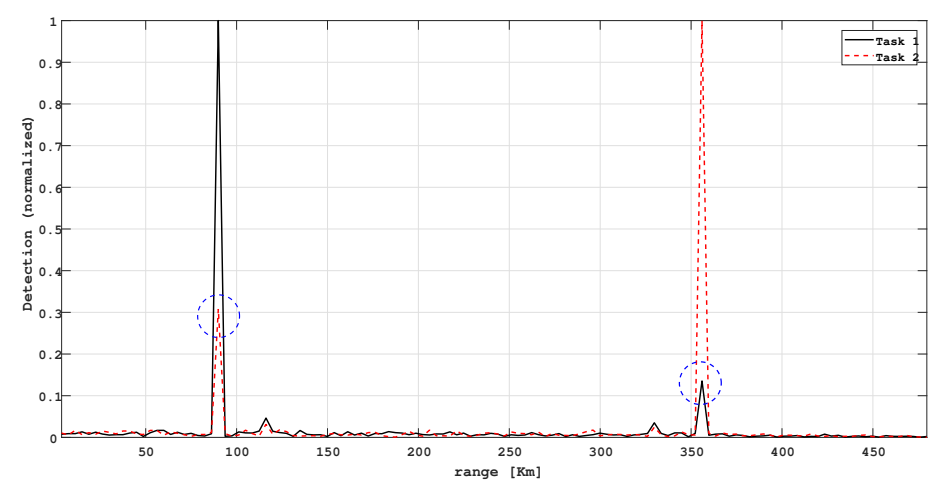

(a) Symbols of Eq. (2.26), major coupling between tasks

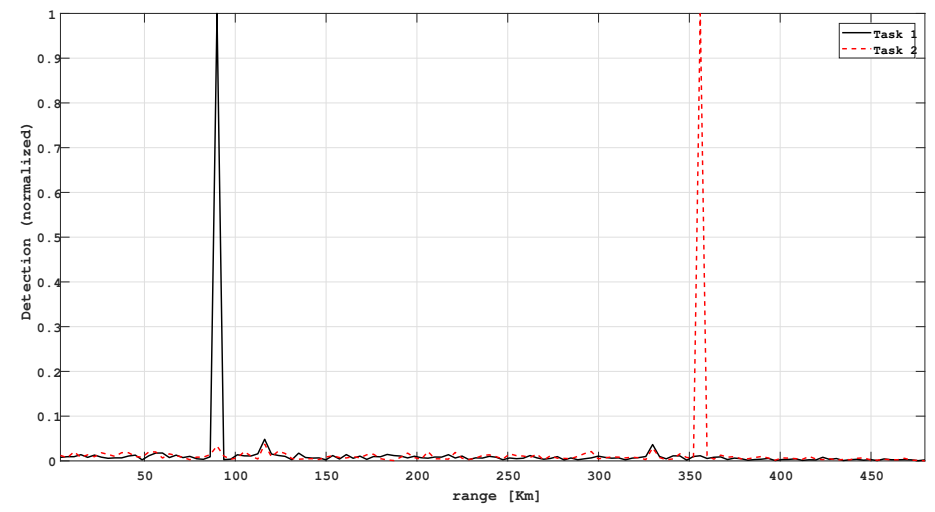

(b) Symbols of Eq. (2.30), effectively decoupled

Figure 2.7: Tracking task of two targets, with different levels of performance accuracy

Then, for ZF to take effect, we need to (partially) re-assign the symbols, as follows:

$$
\begin{aligned}
& \forall k \in K(1): I_{n_{t}}(k, 1)=\tilde{\boldsymbol{a}}\left(\theta_{1}\right)_{n_{t}} \\
& \forall k \in K(2): I_{n_{t}}(k, 2)=\tilde{\boldsymbol{a}}\left(\theta_{2}\right)_{n_{t}}
\end{aligned}
$$

As an example, consider the case in which the two targets are at distinct (polar) locations, relative to the radar, denoted by $\tau_{1} \neq \tau_{2}$ and $\theta_{1} \neq \theta_{2}$. We can now compare the difference that ZF makes, by using the two symbol sets of Eq. (2.26) and Eq. (2.30), in the OFDMA radar parameterized in Table 2.2. We use $\left|r^{(q)}\left(\tau, \theta_{q}\right)\right|$ as the task performance metric.

Illustrated by Fig. 2.7a, the simplistic approach to use corresponding spatial frequency as dedicated data symbols, yields encouraging results. However, the conjecture of data leakage also proves true, so the ZF approach and modifications of Eq. (2.30) is put to test. Fig. 2.7b shows that the new data symbols, rather precisely, focus the transmission of task $q$ to that task only, which makes for a clean and undisturbed task return sequence. So the ZF weights in Eq. (2.28) and Eq. (2.29), decouples the tasks and eliminates their interaction in the transmission signal. We emphasize in this case, the receiver still does beamforming and match filtering. By Zero-Forcing at the transmitters, no energy is sent in the wrong direction.

\begin{tabular}{|c|c|c|c|c|c|c|c|}
\hline Parameter & $K$ & $B_{0}$ & $N_{\mathrm{T}}$ & $N_{R}$ & $d$ & $\lambda$ & $f_{c}$ \\
\hline Value & 128 & $170 \mathrm{kHz}$ & 16 & 16 & $32.61 \mathrm{~m}$ & $92.2438 \mathrm{~m}$ & $3.25 \mathrm{MHz}$ \\
\hline
\end{tabular}

Table 2.2: Parameters for an OFDMA radar without CP, to track two targets 


\subsection{Summary}

In this chapter, we initially developed the basic transmission and reception model, with a task-based approach, for the OFDMA radar. First, at transmitters, complex data symbols for each task are designed and assigned to the allocated subcarrier frequencies for the task. A conventional OFDM signal construction, modulation and demodulation follows. At the receivers, where the arrived signal is deteriorated by clutter, noise and other interfering elements, a scheme is deployed to extract a set of data sequences, most pertinent to each task. The nature of tasks, and the available knowledge to the radar at execution time, control the implementation of this scheme. As proof of concept, we focused on tracking several targets, and interpreted the tracking of each target as a standalone task. Therefore, our OFDMA radar task completion can be assessed by evaluating the expression in Eq. (2.17), from which test statistics can be developed. It is important to emphasize that for each task $q$, a radar input (Eq. (2.13)), and an output (Eq. (2.17)), is developed in the proposed platform.

Then in the chapter, we reviewed common practical issues with the proposed OFDMA radar model in Section 2.1. First, we concluded that adding the Cyclic Prefix guard to OFDM signal, restricts the range extent of the radar. Since we are assuming ideal channel conditions, unless a short range extent is required by our radar, using the CP technique can be skipped. Then, we explored two flexible and efficient solutions, clipping and Selective Mapping, to limit PAPR of OFDM signals within practical constraints. Unfortunately, an analytical relationship between the PAPR threshold (or reduction) was not established in either technique. Thus, the application of both methods appears to follow a trial and error approach.

Moreover, two popular concerns regarding radars were outlined. We developed a simple but powerful scheme to emplace notches in our transmission spectrum, and suppress out-of-band emissions, a situation that can arise in practice quite possibly. Finally, we improved the performance of our task-based radar by introducing Zero-Forcing at the transmitter, which increases the power accuracy, and excludes naive and unnecessary energy dissipation.

So far an OFDMA radar model, with a certain level of practicality, is developed. As highlighted in previously, physical realizability, application convenience, and ease of implementation, are the driving force behind our research on OFDMA technology in radar. So it seems only logical to put all of the methods proposed into test, in order to see the claimed benefits, problems and solutions in action, and approve or refuse some of the conjectures made along the path. Furthermore, at this point there has been very 
little discussion regarding the ocean clutter, communication conditions, and receivers. To be thorough, we must answer questions regarding those areas, which is the focus of the following chapter. 


\section{Chapter 3}

\section{HFSWR Case Study}

In this chapter, we illustrate the efficacy of OFDMA radar. Since this thesis and its underlying research, is in part supported by Raytheon Canada, we develop the OFDMA mode in the context of High Frequency Surface Wave Radar, detecting and tracking ocean targets (mostly ships). An insightful model for this radar is presented in [20] and [21].

One of most important aspects of this radar, is the ocean clutter. We tackle the clutter model, followed by a basic method to decrease its effect, leveraging the frequency flexibility of OFDMA. Also such context helps us to evaluate the methods and techniques described so far, particularly, the constraints on signal power and bandwidth occupancy. As a starting point, let us discover ocean clutter $^{1}$ and its analytical model.

\subsection{Ocean Clutter}

For onshore HFSWR systems, the most prominent interference is the ocean clutter [22]. In radar development, the mathematical representations of sea clutter play a major role. Particularly, the models are used to influence assessment of different radar waveforms, and signal processing algorithms [23]. Moreover, models may often be relied upon as a proxy for actual radar performance, including for acceptance of systems delivery by customers [24]. There is a variety of models, in terms of accuracy and detail; and each one is most suitable to one particular application, of which some examples were mentioned.

Although these models can be developed based on physics of electromagnetic scattering, such knowledge might not be available. So it becomes inevitable to resort to empirical models. Any statistical model in certain aspects relies on empirical data and observations. These experimental representations have their own limitations and scope of application.

\footnotetext{
${ }^{1}$ An alternative term is sea clutter
} 
Therefore, the current statistical models are merely a detailed and insightful guide to a realistic, quantitative assessment, rather than a a reliable prediction for all sea conditions and system scenarios [23].

Robust models to describe ocean clutter, have been developed and they cover a wide range of operating and environmental situations. This enables a reliable analysis of different processing and design techniques in terms of efficacy, and also, generating realistic sea clutter via simulation. In this section, we review a model that fits all this criteria, and explain its integration in our OFDMA radar.

Empirically, a lot of natural factors have been found to partake in characterization of sea clutter. They include the sea $s^{2} \mathrm{te}^{2}$, the wind specifications (speed, duration and direction), and the ocean wave speed. Moreover, some radar parameters influence the measured clutter, such as radar operating wavelength, its bandwidth ${ }^{3}$, the PRF, transmitted power, receiver and transmitter antenna polarizations, and many more [25]. Knowing the values for these parameters, enables the prediction of sea clutter amplitude and phase characteristics, and its temporal and spatial correlation features. The statistical model used to characterize sea backscatter amplitude, is widely recognized to be a firm representation of realistic scenarios [23]. That model is the Compound K-distribution.

\subsubsection{Compound K-distribution for Clutter Amplitude}

Numerous results in the available literature, suggest that the amplitude of sea clutter, matches specific Probability Density Function (PDF). The sea clutter scatter is usually nonGaussian, so the popular choices for PDFs are Weibull, Log-Normal, and K-distribution ${ }^{4}$ [25]. As we discussed, the mathematical model must be capable of uniting the temporal and spatial correlation properties, and that is why compound models, such as Compound K-distribution, are exceedingly appealing.

According to [26], the Compound K-distribution is originated because of the observation that over a wide range of conditions, the clutter backscatter can be modeled well by

\footnotetext{
${ }^{2}$ The degree of turbulence at sea, generally measured on a scale of 0 to 9 according to average wave height.

3 or equivalently, radar range resolution

${ }^{4}$ Currently, no theoretical evidence supports the suggestion that sea clutter follows any of these distributions, and in fact, high fitness to any of the known PDFs is unlikely [23]. However, the representing PDF does not need to be exact.
} 
two components: texture (slow varying), and speckle (fast varying).

$$
\begin{array}{rlrl}
f_{\boldsymbol{X}}(x) & =\frac{b^{\nu}}{\Gamma(\nu)} x^{\nu-1} e^{-b x} & & 0 \leq x \\
f_{\boldsymbol{Y}}(y \mid \boldsymbol{X}=x) & =\frac{1}{x} e^{-\frac{y}{x}} & & 0 \leq y \\
P_{c} & =E[\boldsymbol{X}]=\frac{\nu}{b} & \\
\boldsymbol{Z} & =\sqrt{\boldsymbol{Y}} & &
\end{array}
$$

In the Compound K-distribution, it is assumed that $\boldsymbol{Y}$, the speckle component that models the clutter power, has a conditional exponential distribution of $\boldsymbol{X}$; the component that models the mean clutter power. $\boldsymbol{X}$ is the texture component that follows a Gamma distribution, as seen in Eq. (3.1), in which $\Gamma(\cdot)$ is the Gamma function ${ }^{5}$. Other defining factors are the shape parameter $\nu$ and the power scale factor $b$, and the two are related as in Eq. (3.3), where $P_{c}$ is the measured mean clutter power, and $E[\cdot]$ denotes the expected value operation. Values $b$ and $\nu$ can be deduced from empirical models, which is discussed in appendix A. Finally, the K-distributed sea clutter amplitude, $\boldsymbol{Z}$, is simply achieved by Eq. (3.4).

To integrate the learnings for sea clutter into our case study, we need means to simulate and generate Compound K-distribution data. Since the PDF is rather sophisticated, we cannot use conventional manners to simulate this random distribution. However, [25] gives a closed-form expression for the Cumulative Density Function (CDF) of Compound K-distribution, which enables us to use the Inverse Transform Sampling method [27] to obtain pseudo-random K-distributed numbers. Fig. 3.1 is an illustration of this method, for different values of $\nu$ and unit scale factor $b=1$. However, this approach removes the opportunity to assess the effect of temporal and spatial correlation properties in the radar performance. An alternative way to generate numbers that follow a Compound K-distribution, is to base them upon desired temporal and spatial correlation properties.

Sea clutter observations illustrate that individual cells containing backscatter, generally, are not independent from pulse to pulse. The typical temporal autocorrelation function of sea clutter has a fast drop-off, followed by a slower periodic delay [26]. Therefore, techniques like frequency agility will decorrelate the clutter temporally. The definition for clutter spatial correlation is the cross-correlation between signals echoed from two radially separate sea clutter patches. According to [25], the generic spatial autocorrelation function is the sum of two terms: a peak at the origin, tailed by a scaled clone of clutter

$$
{ }^{5} \Gamma(z) \triangleq \int_{0}^{\infty} x^{z-1} e^{-x} d x
$$




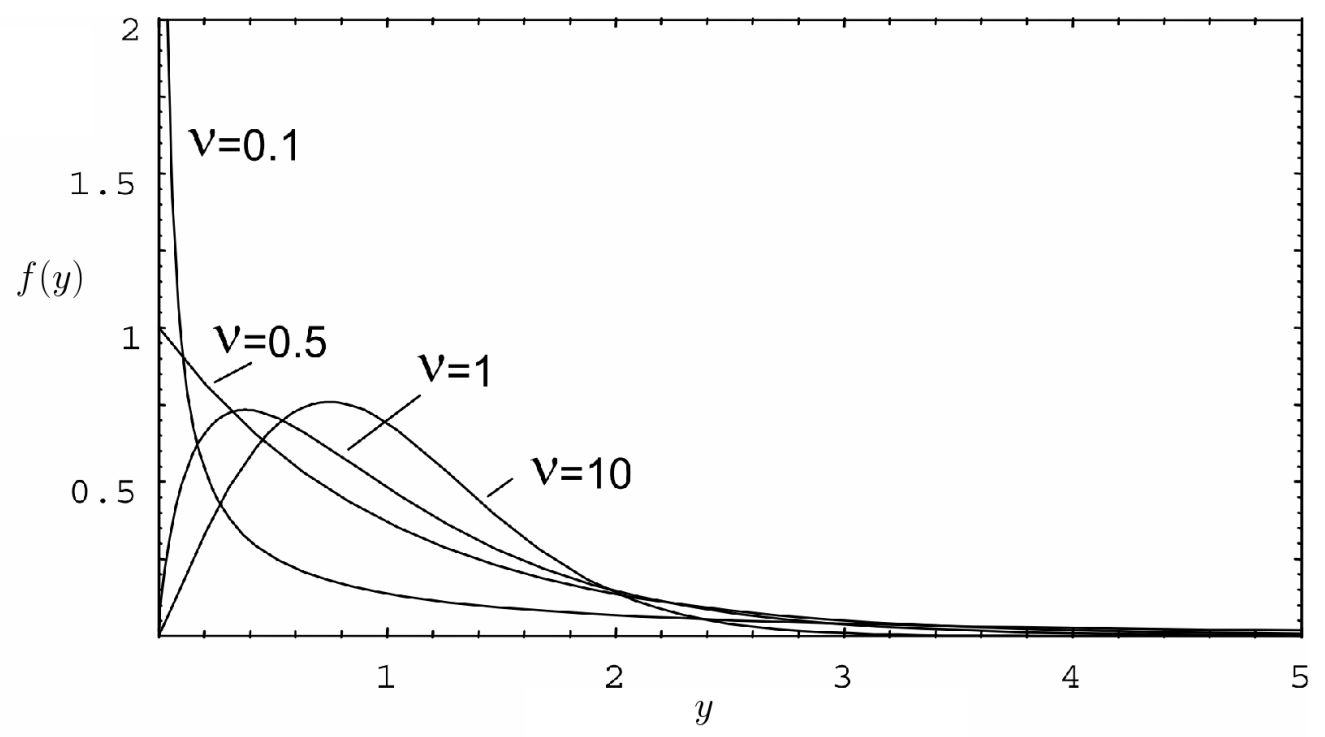

Figure 3.1: Typical PDF of Compound K-distribution, for varying values of $\nu$ and $b=1$. Adapted from [23]

mean reflectivity autocorrelation function. In [28], an estimation procedure for spatial correlation is presented.

There has been numerous attempts to simplify the generation process of Compound K-distribution numbers with given correlation properties [25, 29]. We found the Hybrid Method of [30] to be the most convenient technique for clutter simulation.

The Hybrid Method requires the following input: $\nu, b$, the temporal autocorrelation function $\rho_{T}(\delta)$, and the spatial autocorrelation function $\rho_{S}(\delta)$. The values for $\nu$ and $b$, are calculated based on the radar and natural environment parameters. As a simple choice for these factors ${ }^{6}$, let us assume the sea state 2 to get the values: $\nu=3$ and $b=1.99$. Moreover, in all simulations we need to ensure the autocorrelation functions are realistic. A detailed explanation of the Hybrid Method, as well as the conditions for realizable autocorrelation functions, is presented in appendix B. Our chosen functions are presented in Eq. (3.5), and depicted in Fig. 3.2a and Fig. 3.2b.

$$
\begin{array}{ll}
\rho_{T}(\delta)=e^{-\delta / 10} & \forall \delta \in\{0,1,2, \ldots\} \\
\rho_{S}(\delta)=(0.7+0.3 \cos (0.12 \pi \delta)) e^{-\delta / 12} & \forall \delta \in\{0,1,2, \ldots\}
\end{array}
$$

We compare the results of the Hybrid Method with theoretical Compound K-distribution in Fig. 3.2c, using a Monte-Carlo simulation with 1000 iterations. Then, we obtain the PDF of our generated sequence, based on estimated histogram functions. As shown in Fig. 3.2d, the Hybrid Method generates samples with good fitness to the theoretical

\footnotetext{
${ }^{6}$ in practice, these values are estimated, using maximum likelihood methods developed in [31]
} 


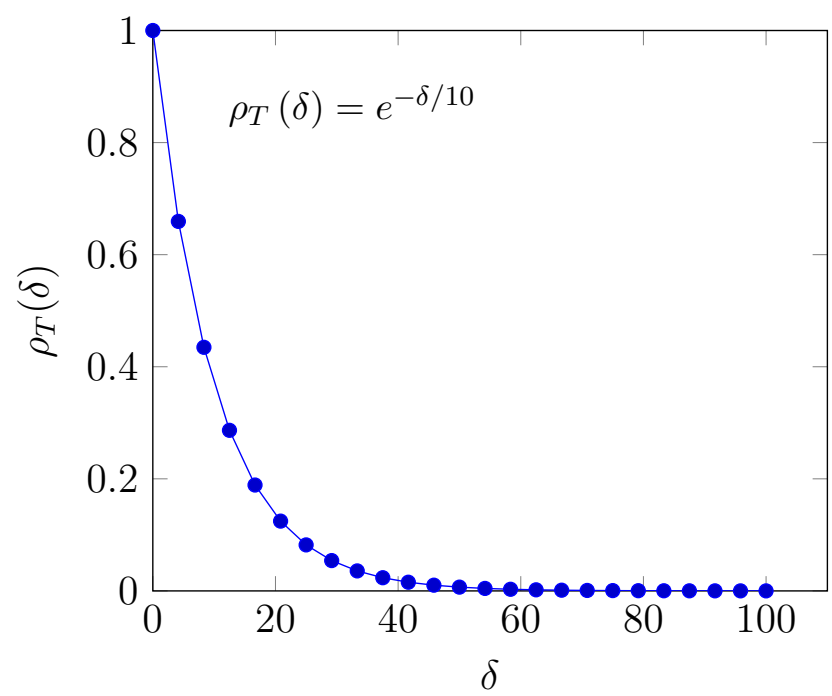

(a) Temporal Autocorrelation

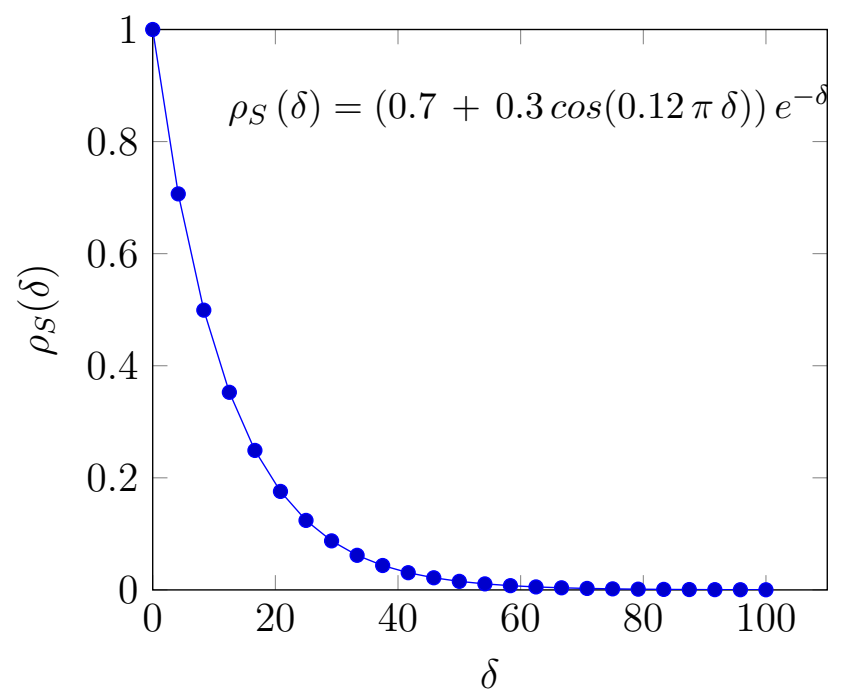

(b) Spatial Autocorrelation

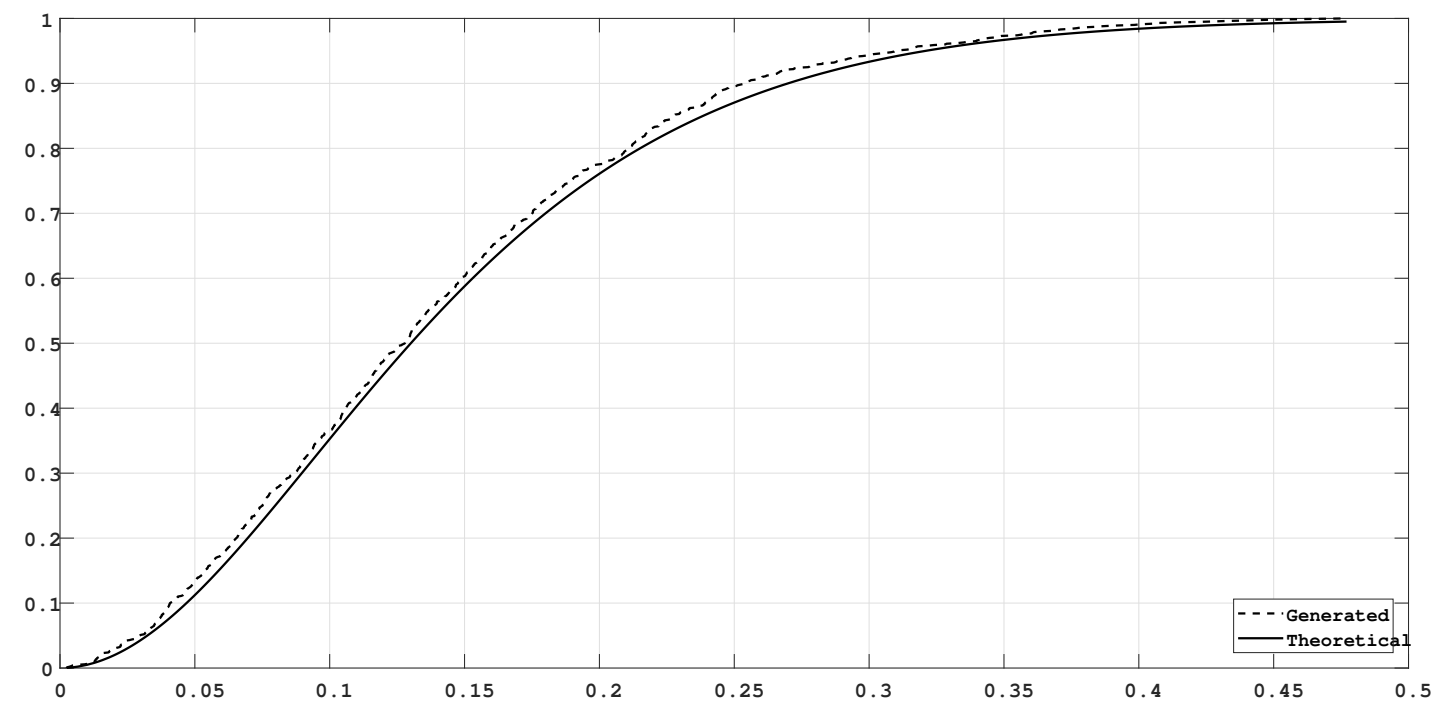

(c) $\mathrm{CDF}$ of theoretical (solid) and generated(dashed) data

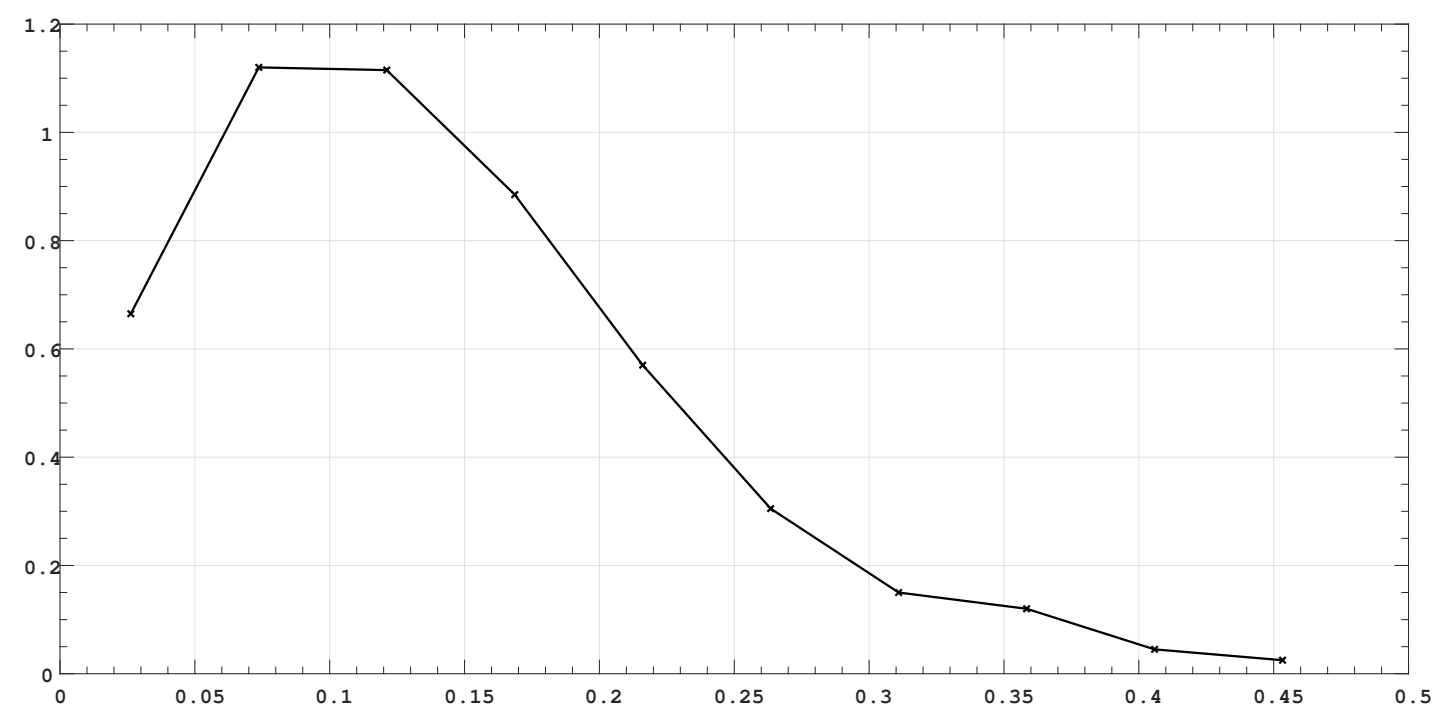

(d) Estimated PDF of data generated with Hybrid Method

Figure 3.2: Using the Hybrid Method to generate sea clutter data 
Compound K-distribution. This confirms that by using the Hybrid Method, we are capable of simulating the sea clutter amplitude with known spatial and temporal correlation properties.

We can now conclude our brief but exhaustive discussion on ocean clutter modeling. In Eq. (2.14), we specified the $c_{n_{r}}(t)$ notation for the effective clutter interference received at each antenna. Let us define that $V$ is the number of clutter patches, with known spatial correlation. For a specific patch, $v$, amplitude is $a_{v}$ generated with the Hybrid Method, and its corresponding range cell is denoted by $\tau_{v}$. Hence, the clutter return term can be defined as:

$$
c_{n_{r}}(t) \triangleq \sum_{v=0}^{V} a_{v} s\left(t-\tau_{v}, \theta\right)
$$

\subsection{OFDMA HFSWR Simulation}

At this point, we have all the ingredients to run a proof of concept simulation, for using OFDMA in radar systems. As mentioned earlier in the chapter, a High Frequency Surface Wave Radar is chosen for this experiment. The relevant values to characterize this system are outlined in Table 3.1, which is the basis of this study. Next, we state the chosen tasks for the radar, and configure the OFDMA signaling scheme based on it.

\subsubsection{Tasks Definition}

For the task-based radar, it is essential to define the tasks at hand for the proof of concept. This topic was briefly touched on in Section 2.3.4. There, we introduced two tasks, each corresponding to tracking of an individual target. In tracking tasks, the most recent distance, azimuth angle, and velocity of that target is known to the radar. Let $R_{1}$ be the distance of target 1 from the radar, and $\theta_{1}$ its azimuth angle. Similarly, $R_{2}$ and $\theta_{2}$ are associated with target 2 . Let task 1 be the tracking of target 1 , and task 2 be the tracking of target 2 .

The HFSWR is asked to track and detect ships as targets, which are $80 \mathrm{~km}$ to $100 \mathrm{~km}$ away from the radar. The system is also most effective in the $-60 \mathrm{deg}$ to $60 \mathrm{deg}$ span of beams $[20,21]$. This enables us to test various target placements here to observe the benefits of OFDMA radar signals. 


\subsubsection{OFDMA Configuration}

The radar range resolution, is tightly related to the transmission pulse width. In that, the signal duration cannot be larger than the corresponding delay to a cell $\Delta R$ away from the radar.

$$
\begin{aligned}
& \Delta R \geq \frac{C}{2 B_{0}} \\
& \Delta R \geq C \frac{T_{\text {pulse }}}{2 K}
\end{aligned}
$$

As stated by Eq. (3.7), the pulse width can restrict the range resolution. Table 3.1 helps us configure the OFDMA signal duration, based on the available bandwidth $B_{0}$ and desired $\Delta R$.

In Section 2.3.1 we showed how the addition of CP to a radar signal, will limit the range extent. However, by the values in Table 3.1, this is not a concern, since to shorten the range resolution in this study, we must have: $T_{p} \geq \frac{T_{\mathrm{o}}}{2}$. That is, the prefix length must be more than half of the signal duration ${ }^{7}$. Although this is theoretically possible, in practice it is considered a design malfunction [15]. Thus, we do not use CP in our system, and thus: $T_{\text {pulse }}=T_{\mathrm{o}}$.

According to Table 3.1, the selected $\Delta R$ is an order of magnitude higher than the theoretical lower bound ${ }^{8}$ enforced by $B_{0}$. This value adds a degree of freedom to chose the $K$ value. Considering the effect of large $K$ values on transmission PAPR, proved in Section 2.3.2, it is best to keep $K$ very close to any lower bound that exists. By choosing $K=512$, the pulse can extend up to a range cell $451.45 \mathrm{~km}$ away, which is greater than the desired range extent. The frequency spacing $\Delta f$ and pulse duration $T_{\mathrm{o}}$, would be automatically determined since our available bandwidth is limited to $170 \mathrm{kHz}$.

Finally, we can select an arbitrary value for number of pulses, $M$, which enables slow-time processing, and makes the clutter temporal correlation properties effective in the OFDMA radar. These chosen values for the OFDMA signalling scheme in a practical HFSWR is summarized in Table 3.2.

\subsubsection{Symbol Assignment}

With the tasks and number of subcarriers defined, the next step is to choose subcarrier subsets for each task, and assign designated task symbols to the subset. In Section 2.3.4

\footnotetext{
${ }^{7}$ or $N_{p} \geq \frac{K}{2}$

${ }^{8}$ the limit is $299792458 \mathrm{~m} \mathrm{~s}^{-1} \div(2 \times 170 \mathrm{kHz})=881.74 \mathrm{~m}$
} 


\begin{tabular}{|c|c|}
\hline Parameter & Value \\
\hline$B_{0}$ & $170 \mathrm{kHz}$ \\
$f_{c}$ & $3.25 \mathrm{MHz}$ \\
$\lambda$ & $92.2438 \mathrm{~m}$ \\
\hline Restricted Bands & $40 \mathrm{kHz}$ to $46 \mathrm{kHz}$ \\
\hline$N_{\mathrm{T}}$ & 16 \\
$N_{R}$ & 16 \\
$d$ & $32.61 \mathrm{~m}$ \\
\hline Range Extent & $400 \mathrm{~km}$ \\
$\Delta R$ & $3.4 \mathrm{~km}$ \\
\hline PAPR & $\leq 3 \mathrm{~dB}$ \\
$\eta_{n_{r}}(t)$ & $\mathcal{C N}(0,1)$ \\
\hline
\end{tabular}

Table 3.1: HFSWR system basic characteristics for the case study

we suggested complex symbols for each of the target tracking tasks, and confirmed as a good guess.

It is evident from Eq. (3.7) that our transmission must leverage all the available bandwidth to preserve range resolution. This is key in any scheme that decomposes the subcarriers into task-specific subsets. After defining the symbols in Section 2.3.4, we opted for an "even vs. odd" configuration; which maximizes the bandwidth of either tasks' subcarrier subset. In other words, when task 1 occupies even-indexed subcarriers, its transmission signal has the bandwidth of $\Delta f \times(K-1)$, and similarly, the bandwidth for task 2 is $\Delta f \times(K-1)$. So, both bandwidths are the maximum possible.

As another supporting example, consider the following scenario: the two targets are next to each other, or $R_{2}=R_{1}+\Delta R$, and the subcarrier subset configuration is "left vs. right", which means task 1 symbols are assigned to the first half of the bandwidth and task 2 symbols occupy the last half. Based on Chapter 2 notation, that is:

$$
\begin{aligned}
& K(1)=\left\{0,1, \ldots, \frac{K}{2}-1\right\} \\
& K(2)=\left\{\frac{K}{2}, \frac{K}{2}+1, \ldots, K-1\right\}
\end{aligned}
$$

This is a flawed subcarrier distribution scheme, as Fig. 3.3b shows. The detection is deteriorates as the OFDMA radar fails to distinguish between the juxtaposed targets. That is because neither task is using as much of the bandwidth as possible. Once this is modified, and the aforementioned "even vs. odd" distribution is applied, Fig. 3.3d illustrates that OFDMA is able to detect two distinct targets. Hence, the task subcarrier 


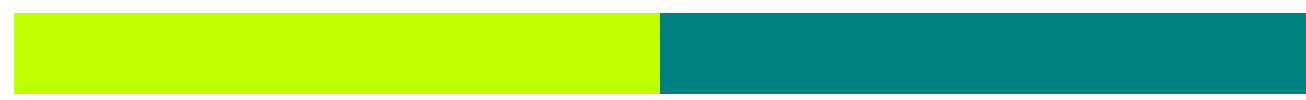

(a) Bad subcarrier assignment

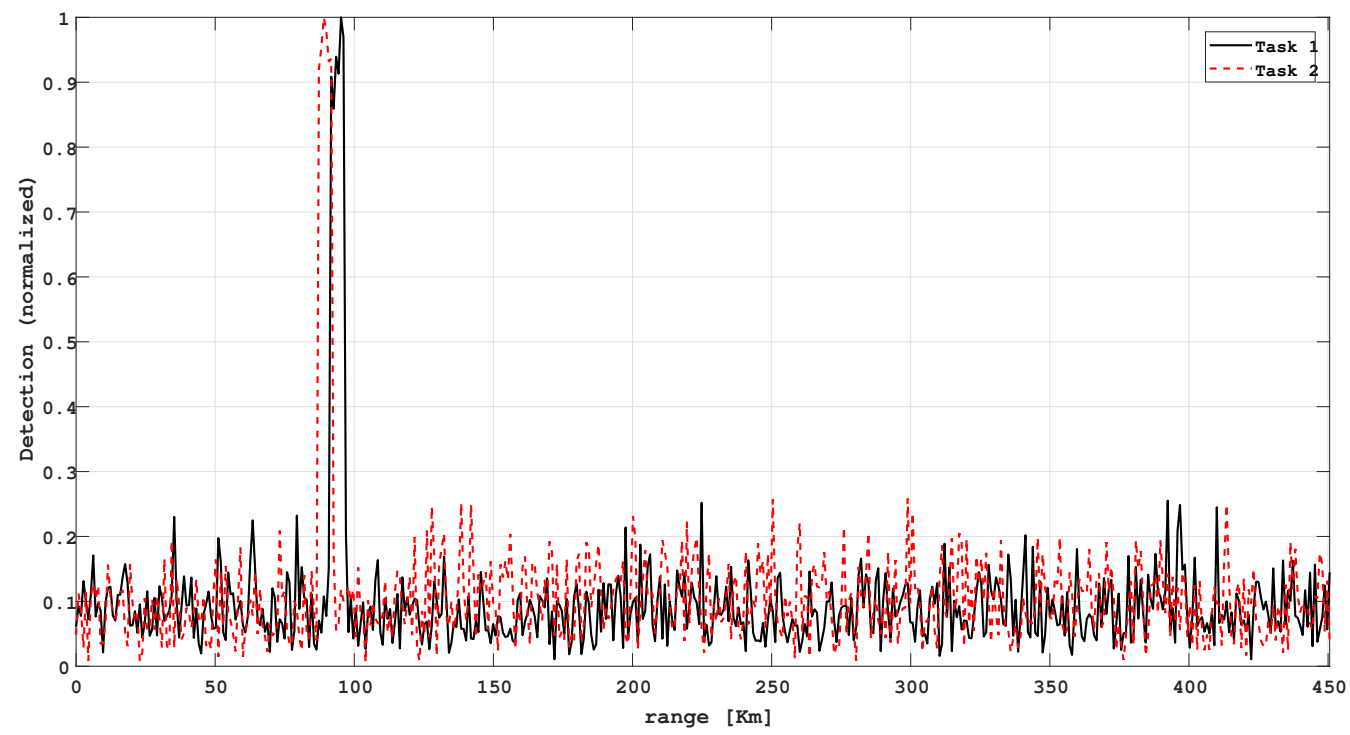

(b) Radar cannot distinguish between two close targets

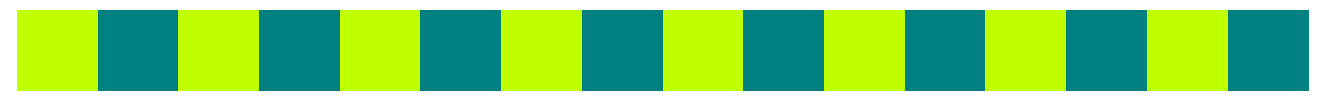

(c) Better subcarrier assignment

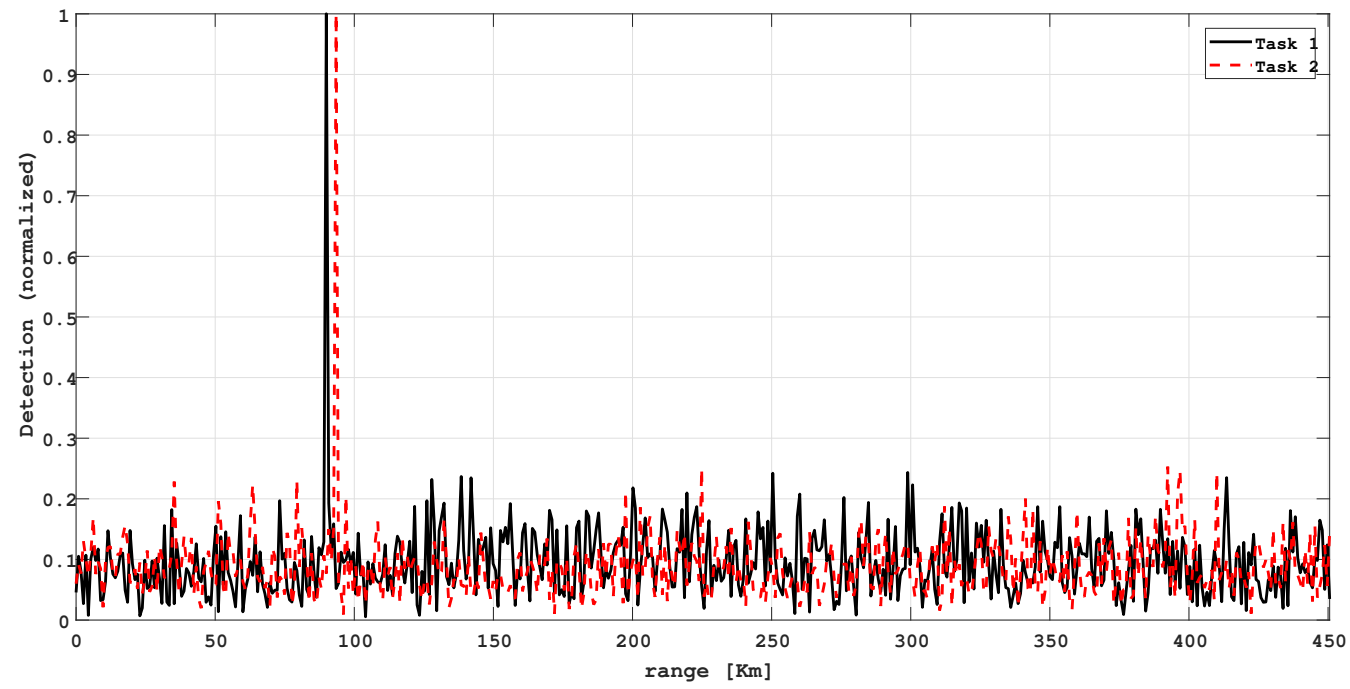

(d) Extended bandwidth corrects the detection

Figure 3.3: Effect of extensive bandwidth on OFDMA radar detection

subsets must all be as widespread in the spectrum as possible.

The topic of bandwidth notches can be worked in by symbol assignment as well. We interpret the notching as a virtual radar task, $q=3$, with its own dedicated subset of 


\begin{tabular}{|c|c|c|c|cc|cc|}
\hline Parameters & $K$ & $M$ & $\Delta f$ & \multicolumn{2}{c|}{$K(q)$} & \multicolumn{2}{c|}{$I_{n_{t}}(k, q)$} \\
\hline \multirow{3}{*}{ Values } & \multirow{3}{*}{512} & \multirow{2}{*}{256} & \multirow{2}{*}{$0.332 \mathrm{kHz}$} & $\mathrm{q}=1$ & $\{0,2, \ldots, 510\} \backslash K(3)$ & $\mathrm{q}=1$ & $e^{j \psi\left(\theta_{1}\right)}$ \\
\cline { 4 - 7 } & & & $\mathrm{q}=2$ & $\{1,3, \ldots, 511\} \backslash K(3)$ & $\mathrm{q}=2$ & $e^{j \psi\left(\theta_{2}\right)}$ \\
\cline { 4 - 7 } & & $\mathrm{q}=3$ & $\{122,123, \ldots, 139\} \cup\{321,322, \ldots, 338\}$ & $\mathrm{q}=3$ & 0 \\
\hline
\end{tabular}

Table 3.2: Final OFDMA radar configuration

frequencies. If transmission is not allowed in some bands within $B_{0}$, we simply put zero as the symbol of corresponding subcarriers. Details for the prohibited bands in the case study are found in Table 3.1.

With that, the OFDMA configuration is complete and presented in Table 3.2. Let us emphasize that all signals are realizable using the streamlined process of Section 2.3.3. Going forward, we account for the band restrictions in the case study.

\subsubsection{PAPR Control}

We already reviewed two prominent techniques to reduce the PAPR in Section 2.3.2. Here we put them to practice, with a $3 \mathrm{~dB}$ limit to this metric. Without either of clipping or SLM control in place, this constrain is not satisfied among several transmitters. Of course, lack of transmission power in subcarriers of $K(3)$ (restricted frequencies), effectively decreases the PAPR.

Previously, we discovered that no analytical relationship exists between the PAPR threshold and the reduction system parameters. Thus, we are forced to use trial and error. We designed Algorithm 1, in which iteratively the clipper bound on high PAPR signals is tightened until they are below $3 \mathrm{~dB}$. For the SLM method, the only parameter to tweak would be $U$, the number of data symbol clones to search among for the lowest PAPR. To gauge the efficacy of all techniques, we test them in a scenario where the two targets are well separated ${ }^{9}$. We execute the SLM method for two values of $U$, as described in Table 3.3.

Fig. 3.4 shows some performance deterioration when PAPR is restricted, however, this effect does not hinder task completion in any case. To choose the best technique, we can consider the fact that SLM is comparatively computationally heavy, and it requires transmission of side information for full data retrieval at the receivers. Therefore, in our HFSWR system, the clipping method of Algorithm 1, is proven to be efficient and satisfactory to control PAPR with.

This concludes the topics on transmission. In the following sections, we see how

\footnotetext{
${ }^{9}$ this is necessary to have distinguished task specific symbols
} 


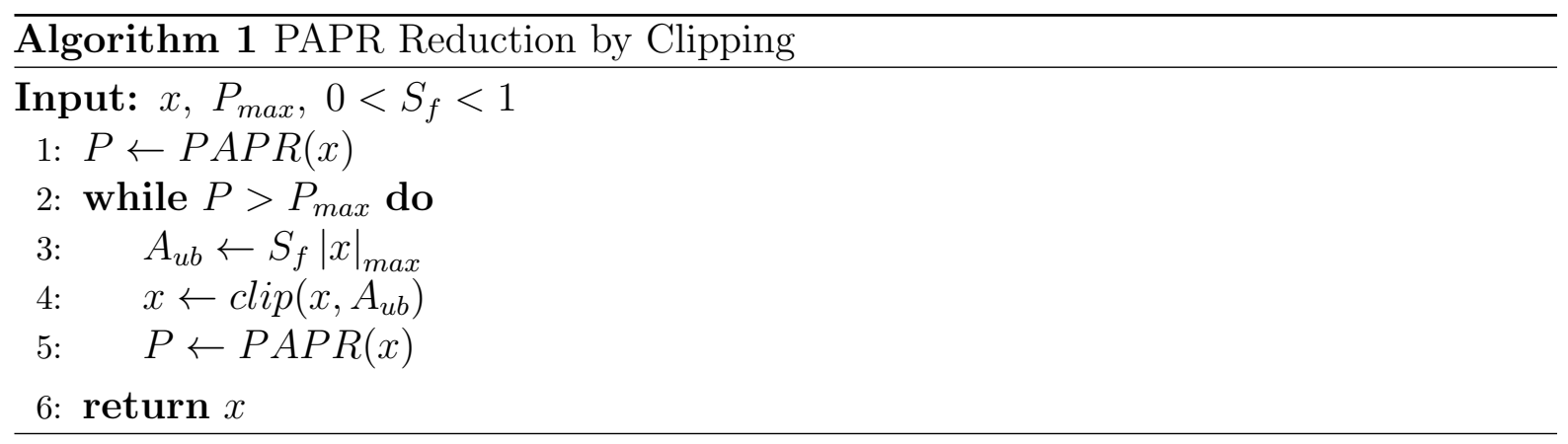

OFDMA can transcend task performance, and ocean clutter can be battled by an adaptive symbol assignment scheme.

\subsubsection{Target Resolution of OFDMA Signals}

In order to better see the benefits of using OFDMA methods in radar, we challenge the developed system here in difficult scenarios. To this end, we incorporate the clutter model developed in Section 3.1 to our examples, with shape factor $\nu=3$ and scale factor $b=1.99$. The clutter correlation properties are expressed in Eq. (3.5).

We can see in Fig. 3.5 that, in presence of noise and clutter, performance of task 1 improves as the number of subcarriers increases. This is expected since the transmission becomes more diverse in frequency and has a higher power. However, the cost might be too high, as the notch filtering process and clipping procedures become more sophisticated and too heavy to compute. Therefore, the system implementation would limit the reasonable choices for $K$. Using $K=512$ seems logical, since it enables a radar range extent of $400 \mathrm{~km}$, and as illustrated in Fig. 3.5 it performs comparatively similar to higher values of $K$ in adverse communication conditions

Another challenging scenario for the radar, would be when it is asked to track two targets that are close in distance, or azimuth angle, or both. We judge the task performance based on angle specific versions of received signals, shown in Eq. (2.17). Hence, the lack

\begin{tabular}{|c|ccc|c|}
\hline Method & $\begin{array}{c}\text { Min. } \\
\text { Reduction }\end{array}$ & $\begin{array}{c}\text { Average } \\
\text { Reduction }\end{array}$ & $\begin{array}{c}\text { Max. } \\
\text { Reduction }\end{array}$ & $\begin{array}{c}\text { Computation } \\
\text { Load }\end{array}$ \\
\hline Clipping & $0.733 \mathrm{~dB}$ & $2.282 \mathrm{~dB}$ & $3.226 \mathrm{~dB}$ & Medium \\
SLM $(U=4)$ & $0.907 \mathrm{~dB}$ & $2.591 \mathrm{~dB}$ & $3.413 \mathrm{~dB}$ & High \\
SLM $(U=6)$ & $0.753 \mathrm{~dB}$ & $2.132 \mathrm{~dB}$ & $3.185 \mathrm{~dB}$ & High \\
\hline
\end{tabular}

Table 3.3: Implementation comparison of different PAPR reduction techniques 


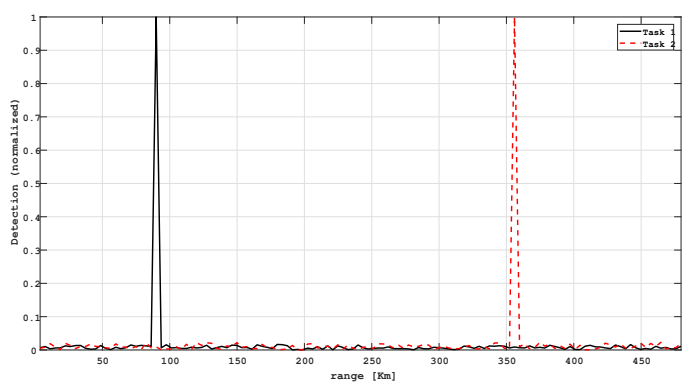

(a) No papr reduction

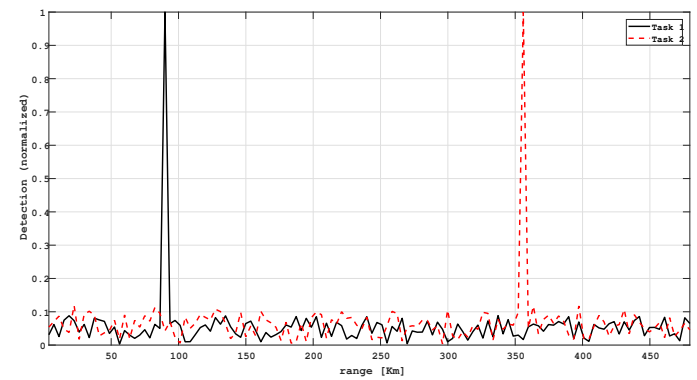

(c) PAPR reduced by $\operatorname{SLM}(U=4)$

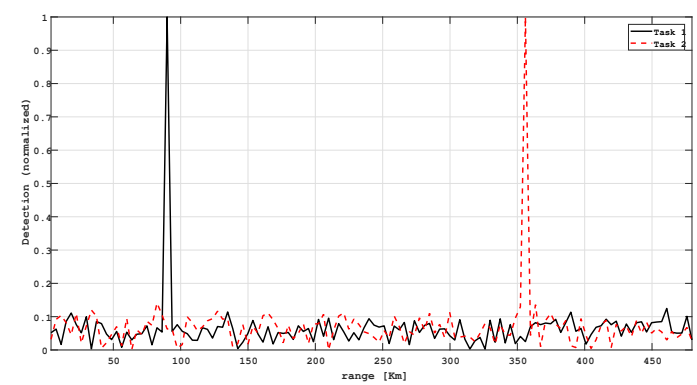

(b) PAPR reduced by Algorithm 1

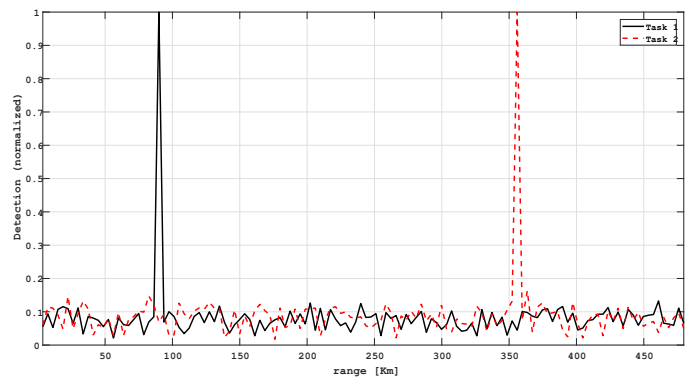

(d) PAPR reduced by $\operatorname{SLM}(U=6)$

Figure 3.4: Task performance comparison of different PAPR reduction techniques

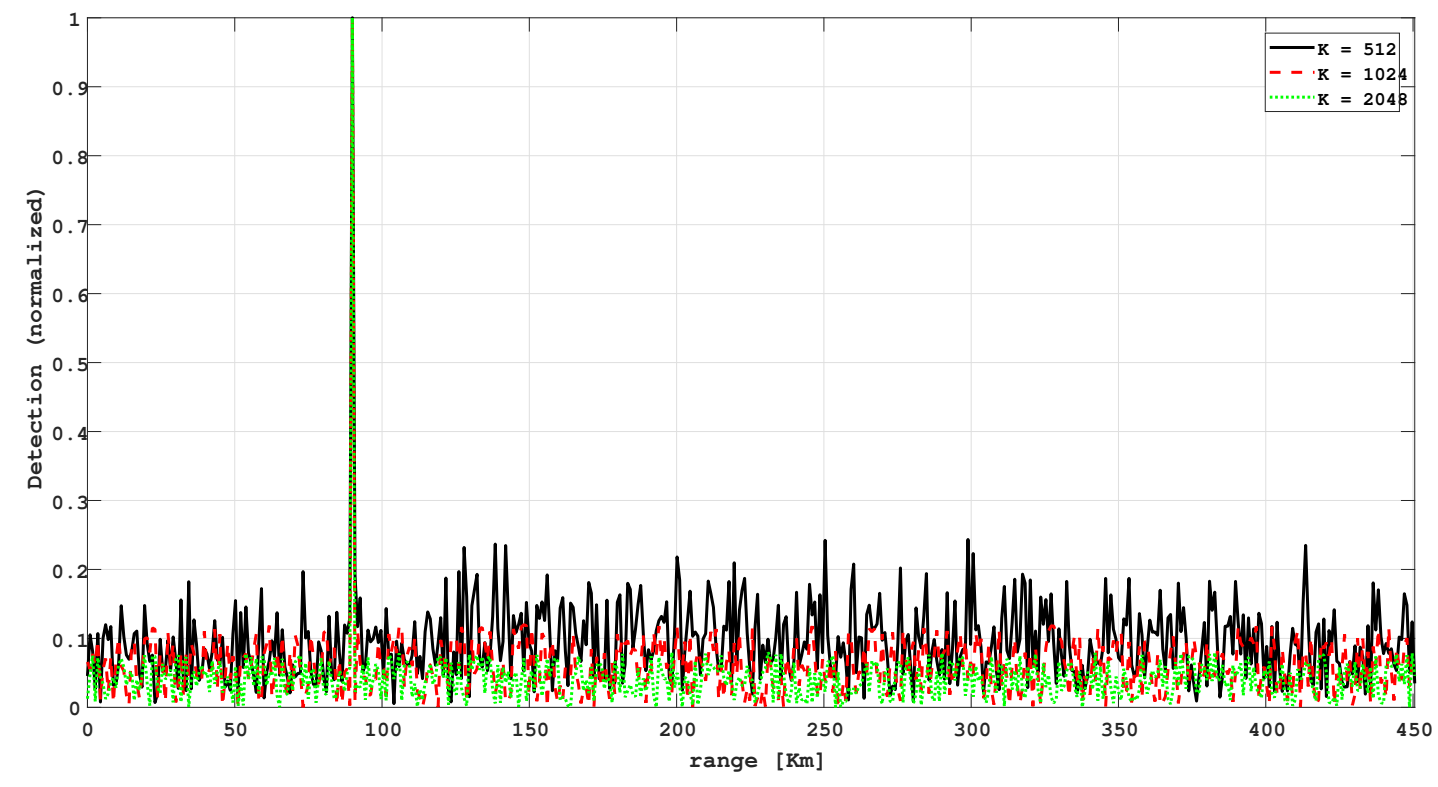

Figure 3.5: Individual task performs better with higher number of subcarriers: $K=$ $\{512,1024,2048\}$ 
of rang or angle resolution, would result in false detection of either target.

Let us examine the case where the two targets are at the same azimuth angle (relative to the first receiver). Fig. 3.6a illustrates the angle resolution of OFDMA radar in tracking the targets. Evidently, both targets are tracked accurately, which means sufficient angle resolution exists in the framework as a result of task specific symbols used in the transmission.

Another difficult case is to track the two close targets in terms of distance, which means $\left|R_{1}-R_{2}\right|=\Delta R$, with $\Delta R$ being the given radar range extent. We see in Fig. 3.6b that the high number of subcarriers, provides a reasonable range resolution in this scenario as well. Of course we expected this, as $K$ was selected based on $\Delta R$.

Previously we illustrated how OFDMA offers a flexible framework to design radar signals, within practical limits. Here we outlined that such framework, pledges a reasonable range and azimuth resolution. Perhaps this would motivate a deeper look into ways of introducing techniques from OFDMA wireless systems into radar platforms.

\subsubsection{Ocean Clutter Rejection}

This chapter started with a close look on modeling the ocean clutter for the proposed OFDMA radar framework. There we introduced the means to simulate the clutter for the radar. The clutter simulation parameters should not be tweaked too freely, as we established earlier that the shape and scale factor of the underlying Compound Kdistribution are determined according to radar characteristics and environmental factors.

In [25], some realistic and measured values for $\nu$ are reported. There, it is observed that a bigger $\nu$ is often associated with a more disturbing clutter. Theoretically, higher values of $\nu$ means higher probability of stronger clutter echoes, illustrated in Fig. 3.1. This supports the claim that spikier clutter is associated with larger $\nu$. As seen in Fig. 3.7, spikier clutter is associated with increased sidelobe levels in the task returns of our OFDMA radar. For convenience, we opt for a friendly clutter behaviour, expressed by $\nu=3$. Moreover, the clutter scale factor is $b=1.99$, and its correlation properties are stated in Eq. (3.5).

To achieve better task completion, we now propose a clutter rejection method, based on frequency decomposition of the clutter returns angled towards each target. In this OFDMA simulation, we can see the frequency distribution of $c_{n_{r}}(t)$, on each subcarrier.

The chosen temporal correlation of the clutter, Fig. 3.2a, suggests that clutter returns at later pulses are virtually uncorrelated ${ }^{10}$. So to gauge the clutter frequency decomposition,

\footnotetext{
${ }^{10}$ this assumption is true in practice, based on observations. See $[25,24,26]$
} 


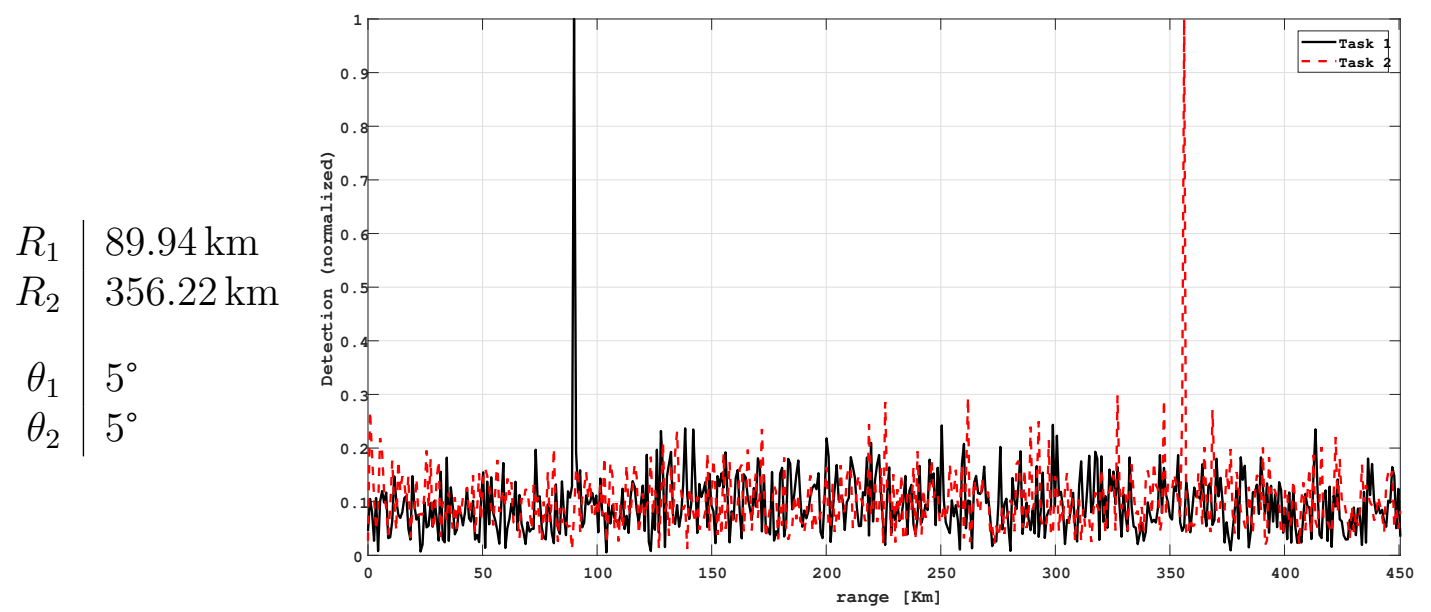

(a) Target tracking in similar azimuth positions

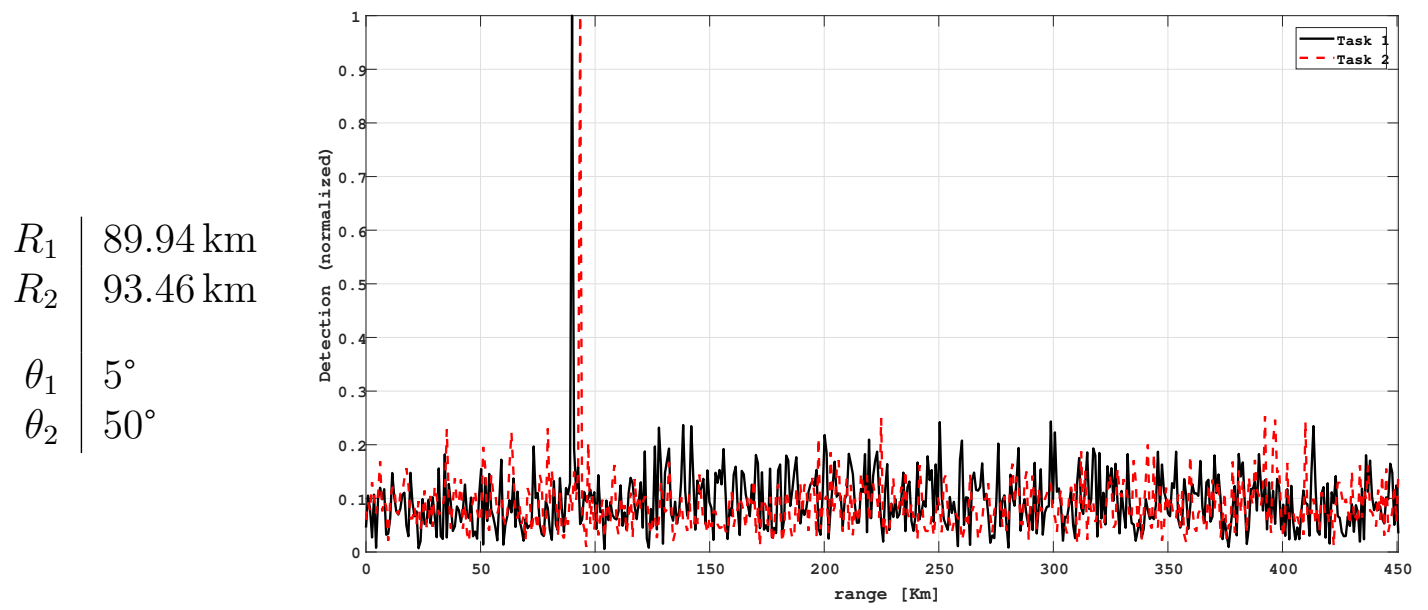

(b) Target tracking in close range cells

Figure 3.6: Benefits of OFDMA for closely juxtaposed targets tracking

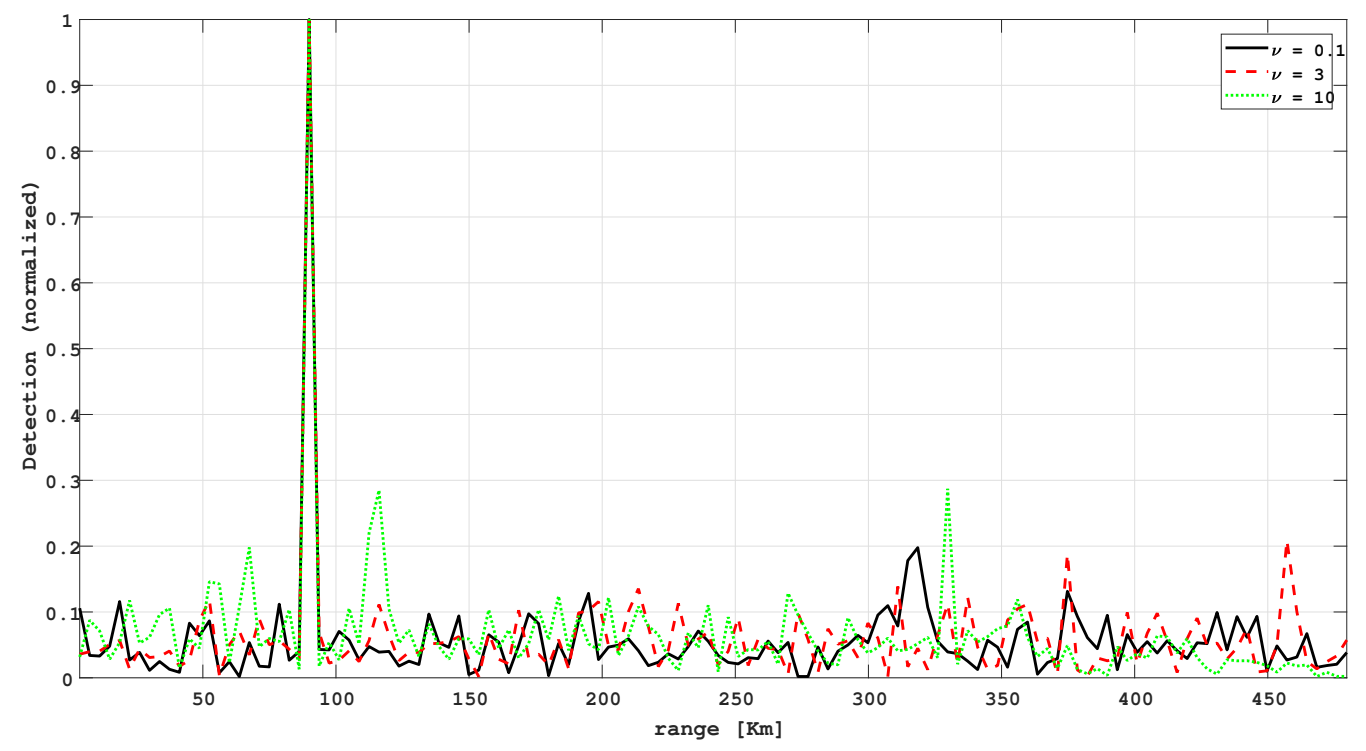

Figure 3.7: OFDM signaling can handle spikier clutter, for $\nu=\{0.1,3,10\}$ 


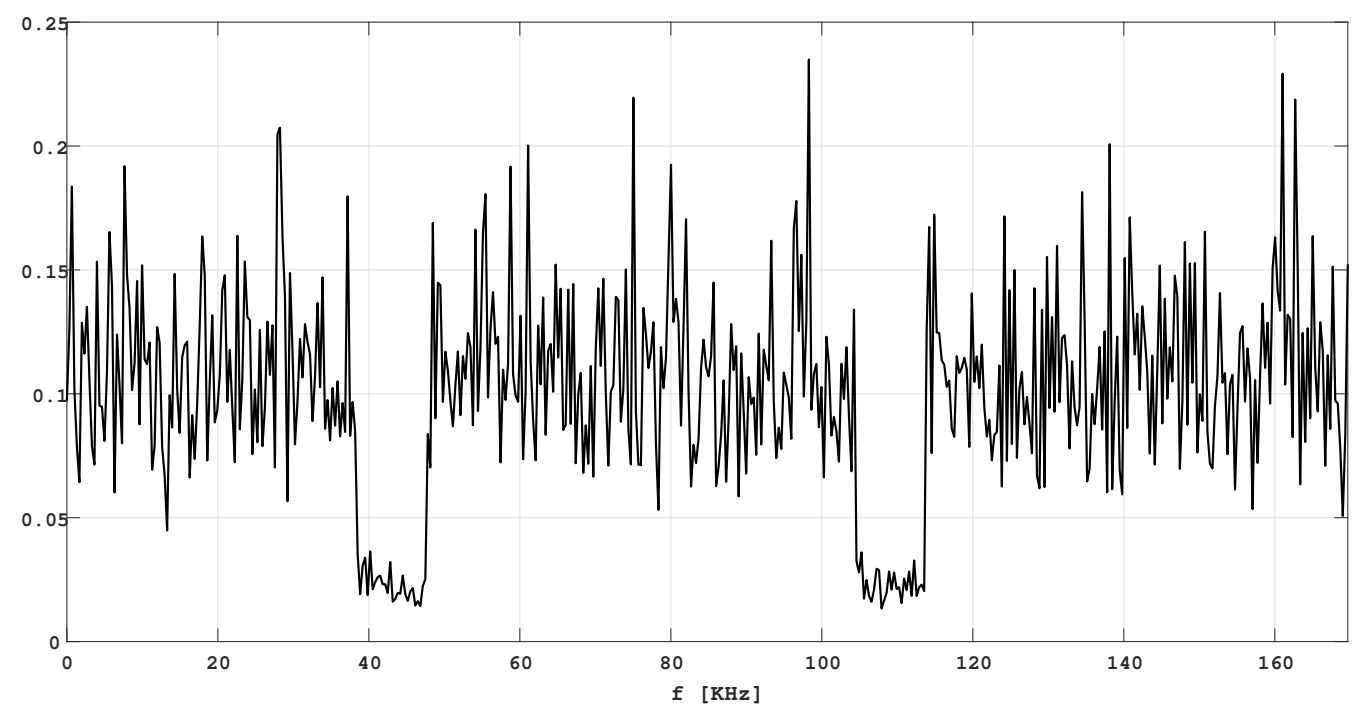

Figure 3.8: FFT of correlated clutter returns

we must examine the pulse returns in small batches, where the signals in each batch is correlated. For instance, the $K$-point Fast Fourier Transform (FFT) of clutter returns in the first 64 pulses, is illustrated in Fig. 3.8 .

This observation is motivation to plant a procedure to obtain knowledge regarding the clutter prior to task execution, and use it to improve the radar performance. Let us transmit radar signals, in batches of size $M=64$, that only look at either $\theta_{1}$ or $\theta_{2}$. Then we examine $C_{q}(f)$, the $K$-points $\mathrm{FFT}$ of the received signals. If at certain frequency bins, the spectral density is strong, we hazard the guess that it might be due to the clutter. Hence, if transmission is nulled at those subcarriers (bins), the clutter might be suppressed. By the same token, if some frequencies outside the restricted bands, show weak power, then the clutter effect might be absent there and hence, they should prioritized in our symbol assignment. We emphasize that this approach is a proof of concept only. To illustrate this, we assume the targets to be well apart both in distance and angle. That is $R_{1}=89.94 \mathrm{~km}, \theta_{1}=5^{\circ}$ and $R_{2}=356.22 \mathrm{~km}, \theta_{2}=50^{\circ}$. We also define $\xi$, a tolerance ratio to distinguish strong or weak values in the FFT of reception. In other words, if the transmission is angled to $q$-th task:

$$
\begin{gathered}
E_{c} \triangleq \frac{1}{K} \sum_{k=0}^{K-1}\left|C_{q}\left(f_{k}\right)\right| \\
\forall k \in\{0,1, \ldots, K-1\}, 0<\xi<1:\left\{\begin{array}{l}
\left|C_{q}\left(f_{k}\right)\right| \geq(1+\xi) E_{c} \Rightarrow \text { remove } f_{k} \text { from } K(q) \\
\left|C_{q}\left(f_{k}\right)\right| \leq(1-\xi) E_{c} \Rightarrow \text { append } f_{k} \text { to } K(q)
\end{array}\right.
\end{gathered}
$$




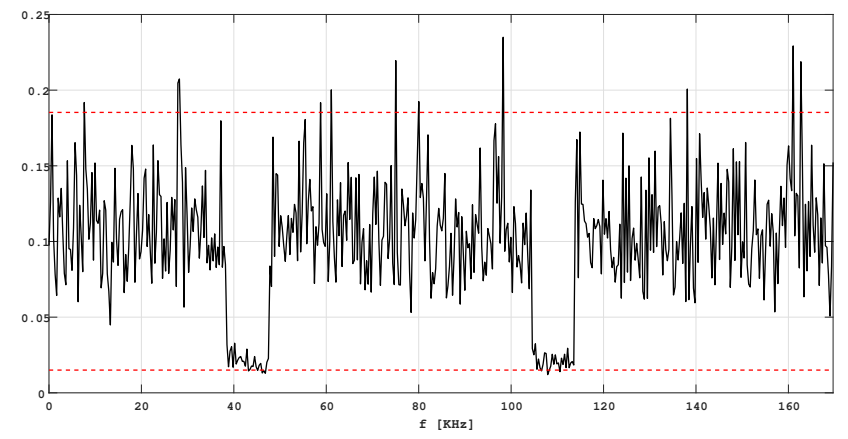

(a) Clutter returns when looking at $\theta_{1}$

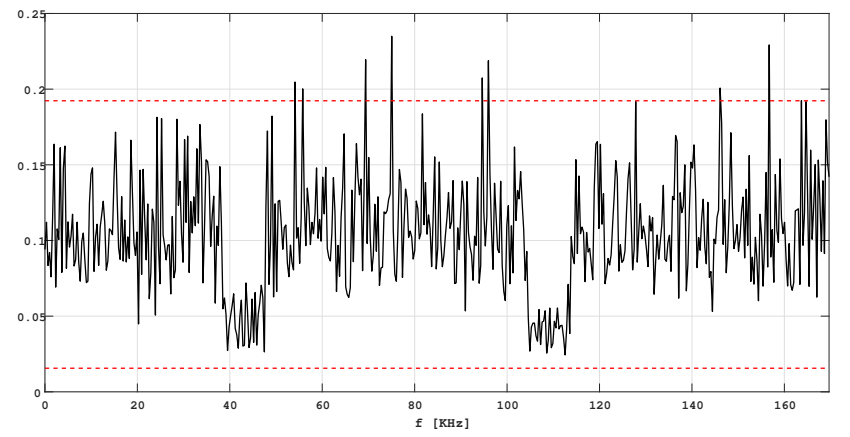

(b) Clutter returns when looking at $\theta_{2}$

Figure 3.9: FFT of angle specific clutter returns, with $\xi=0.85$

In Fig. 3.9, we see the thresholds are set $85 \%$ above and below the mean of the target specific clutter returns, $\left|C_{1}(f)\right|$ and $\left|C_{2}(f)\right|$. As illustrated, in this setting, the clutter affects target 2 , and there are few frequency bins that need to be removed from $K(2)$ and no additional bins can be introduced, since the entire spectrum is above the lower threshold. As for Fig. 3.9a which depicts the specific returns beamformed to target 1 , the only subcarriers associated have low magnitude, belong to restricted bans (or $K(3)$ ), therefore, they would not be added to $K(1)$. Much like $C_{2}(f)$, some of the symbols need to suppressed here too. Overall, in this example, the only possible improvement in performance would be from nulling transmission on frequencies that are most interacted with the sea clutter. Of course, for a tighter tolerance (lower $\xi$ ), the arrangements would be different.

Both Fig. 3.9a and Fig. 3.9b show some of candidate bins for removal, sit at the end of the bandwidth. In Section 3.2.3 we observed the importance of preserving the bandwidth associated to each task, as extended as possible. Thus, any rearrangements to either $K(1)$ or $K(2)$, must be deliberate not to shrink the bandwidth unnecessarily. By applying these considerations to our symbol assignment, we can see a slight but evident improvement in radar performance. Fig. 3.10 shows the tracking tasks after deploying clutter rejection, in which we zoomed in on the sidelobes for clarification. In Table 3.4, a summary of added and removed subcarriers, and the change in Peak to Integreated Sidelobe Ratio (PISR) level for each task, is presented.

\begin{tabular}{l|c||c} 
& Task 1 & Task 2 \\
\hline $\begin{array}{l}\text { Removed } \\
\text { Added }\end{array}$ & $\{84,184,226,296,416,490\}$ & $\{163,209,285,289,493\}$ \\
PISR & $\emptyset$ & $\emptyset$ \\
& 1.3814 to 1.4114 & 1.2349 to 1.2879
\end{tabular}

Table 3.4: Clutter rejection effect on sidelobes 


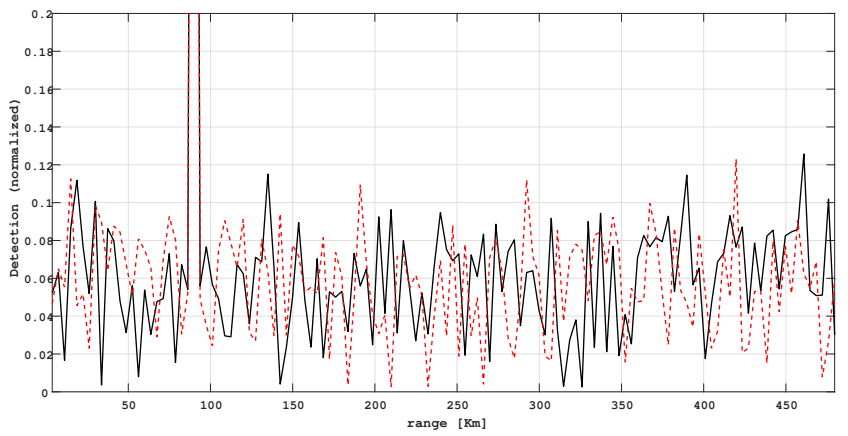

(a) Task 1 Sidelobes

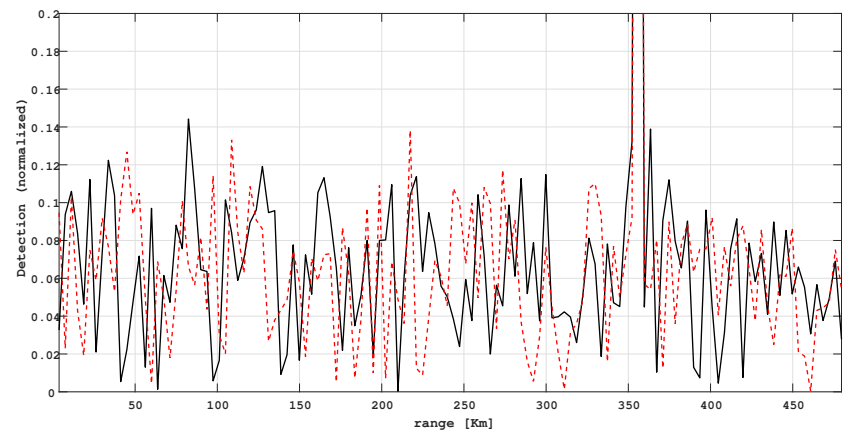

(b) Task2 Sidelobes

Figure 3.10: Task return sidelobes before (solid) and after (dashed) removing strongly clutter affected bins from symbol assignments

\subsection{Summary}

After developing a practical OFDMA radar framework in previous chapters, we applied the lessons in the context of High Frequency Surface Wave Radar built by Raytheon Canada. This introduced a new set of challenges, particularly the interference of ocean clutter.

In addition to the overview of current literature on ocean clutter, as well as mathematical and empirical efforts to model it, this report also explained the Hybrid Method, an efficient and comprehensive simulation technique to generate ocean clutter samples, for the purpose of radar performance assessment, and predicting possible practical scenarios and solutions to them. Consequently, in this chapter, we proposed a rudimentary clutter rejection method, based on the OFDMA radar framework developed earlier.

Furthermore, we concluded benefits of OFDMA singals with regards to range and azimuth resolutions. We also managed to satisfy practical contraints, using the common solutions to similar issues in OFDM communications, such as PAPR reduction techniques. The streamlined interpolation and decimation method to make signals physically realizable, introduced in Section 2.3.3, was also confirmed as a solid mean to apply band restrictions to radar transmission.

As for unpromising discoveries, it appeared that integrating OFDM into radar waveform design, would grow exponentially in difficulty once the number of tasks increases. All of the remarks in this chapter were made based on two tracking tasks, which in practice contains a small portion of radar functionalities. Therefore, a comprehensive review of the objectives, contributions, and reservations unearthed in this correspondence is necessary. 


\section{Chapter 4}

\section{Conclusions}

\subsection{Review}

The focus of this thesis is the development of a High Frequency Surface Wave Radar using Orthogonal Frequency-Division Multiple Access to execute multiple tasks simultaneously, with practical functionality and desirable characteristics.

The rationale behind combining OFDM signal processing techniques and radar, can be categorized in many different levels. It is known that frequency diversity in radar transmission, imporves target detection. Moreover, OFDMA offers waveform design in the digital domain which is highly convenient and flexible for implementation. Data transmission on orthogonal subcarriers, would enable us to decompose radar signals in the frequency domain, and assess their behavior and make adjustments accordingly. The orthogonality also reduces possible channel adversities, such as multi-fading and intersymbol interference. Finally, unrelated information can be weaved into the transmission and fully retrieved at the reception, which on a fundamental level, instigates the collaboration and co-existence of radar and commercial wireless systems on the same bandwidth.

The OFDM method spreads the signal throughout the available spectrum, and provides adjustable carrier allocation with better efficiency than conventional radar signal design schemes, such as phase coding [2]. Thus, once we interpret radar systems as executor of several tasks, in which each task requires its designated signal transmission and frequency allocation, a task-based radar communication scheme is developed, capable of interleaving tasks within one another in transmitters, and extract relevant information from arrived echoes in receivers. In this report, we proposed an OFDMA radar system which is capable of performing multiple tasks simultaneously, while following the practical standards and constraints of conventional HFSWR systems.

There are several constraints on HFSWR in practice, as the system is at the high 
end of commercial radars in terms of cost and maintenance. Thus, it is important to be able to restrict the transmission a HFSWR when necessary. Furthermore, all signals propagated from the system must be physically realizable, in order to decrease chances of depreciation and malfunction. This also limits power characteristics of the signal below a determined threshold.

In the realm of surface wave radars, one of the most prominent concerns is the interference caused by ocean clutter. There has been enormous effort in modeling the sea clutter based on observations and measurements, dating back to the early 80s [32]. The clutter nature depends strongly on environmental factors, such as the sea state, wave and wind characteristics. Additionally, the radar transmission properties also plays a prominent role in the clutter disturbance intensity. Generally, an accurate representation of sea clutter for each and every scenario cannot be achieved, so researchers resort to the Compound K-distribution for statistical modeling of clutter amplitude, whose parameters can be estimated based on measurements from a radar system in presence of the clutter. Finally, slow and fast varying components of the clutter are modeled as temporal (pulse to pulse) and spatial (range to range) correlations. These findings facilitate the simulation of clutter data for purposes of radar performance assessment and prediction.

\subsection{Contributions and Discussions}

In this paper, we proposed task-based OFDMA radar signal transmission and reception model, by treating radar tasks similar to users in wireless systems. As a proof of concept, we defined two target tracking tasks. In tracking functions, the radar has a recent knowledge of targets locations, based on which we chose designated data symbols for each task.

In order to utilize the bandwidth to full capability, we emphasized an awareness of allocating subcarrier subsets to each task with the maximum bandwidth possible. However, using several subcarriers to comprise a signal, inherently increases the Peak-to-Average Power Ratio, which is a practical concern. We showcased that existing PAPR reduction techniques in wireless communications, can also be deployed in radar systems with similar results.

Another issue with OFDM signals is the possible intersymbol overlap which in turn corrupts the data at the receivers and hinders full retrieval and therefore, system performance. One of the means to suppress the probability of this, is the Cyclic Prefix guard intervals. We investigated applying $\mathrm{CP}$ to radar signals, to conclude that $\mathrm{CP}$ brings an upper bound to radar range extent. Furthermore, when transmitting the signal comprised 
of subcarriers to both tasks, we observed a leakage of energy between the two tasks, which could not have been prevented with CP. We used a ZF approach to eliminate coupling between tasks. The extension to several tasks is also trivial. ZF is applied at transmitters.

Lastly, we configured our OFDMA radar to follow the practical standards and constraints of a HFSWR system instance. The benefits of using OFDM signal models included high performance accuracy, interference and clutter resilience, and spectrum adaptability. We also demonstrated the efficacy of methods that address PAPR issues and nulled frequency transmissions. The OFDMA framework also allows us to optimize the symbol design process to obtain adaptive waveforms that reject clutter. To showcase this, we developed a fast and extensive simulation software to generate ocean clutter data for any sea environment. One of the most notable merits of this clutter simulation framework, was the capability to analyse the clutter effect throughout the transmission bandwidth. Using this feature, we tested a clutter rejection procedure in which for each target, subcarriers that are strongly affected by clutter are identified and transmission is disabled on them. Additionally, the subcarriers that are least interacted by clutter are added to the task based transmission frequencies. This marginally improved the tracking performance, promising the possibility of fresh approaches towards clutter rejection.

\subsection{Future Work}

The proposed task-based OFDMA radar, illustrated exciting results, and confirmed most of the conjectures regarding its functionality and benefits. However, at the current state, the scope of the radar tasks is very limited. Thus, an interesting extension to this research would be designing a streamlined symbol identification and assignment process based on the tasks. Then we can relax the condition of knowing recent position, azimuth and velocity of targets. In the case of surveillance tasks, what correspondence should exist between the region of interest and the task related subcarrier symbols.

Furthermore, in commercial OFDMA systems, the transmission is extremely optimized based on the user request. Here we assumed a uniform power distribution between tasks, which we suspect might not be an optimal solution, similar to wireless communications [33]. The optimization would fit nicely with current transmission power distribution techniques in radar as well, but using the OFDMA we can attune the process to leverage the full bandwidth capacity.

Finally, it appears that the radar and communications industry, are exceedingly interested in building co-existing and collaborating systems [7]. OFDM seems to be the only chosen avenue for this realization. However, the approach is predominantly 
integrating radar capabilities into wireless systems. We sense the results outlined in this report, make a strong case for the reverse approach, which is to explore the possibility of adding digital communications and information transfer to an existing OFDMA radar. 


\section{Appendix A}

\section{Empirical Model for Clutter Scale Factor}

The clutter scale factor, $b$, is mainly responsible for the power characteristics of received clutter echoes. The relationship is inverse, that is, the lesser values of $b$ correspond to more powerful reflections from the sea surface. The scale factor can be predicted from a knowledge of cutter shape parameter $\nu$, clutter reflectivity, and radar characteristics. This prediction is presented in Eq. (A.1) whose variables are summarized in Table A.1.

$$
b=\frac{\nu}{P_{t} G_{t}^{2} \frac{\lambda^{2} f^{4}}{(4 \pi R)^{3}}\left(\sigma_{0} \theta_{B} C \frac{\tau_{\text {pulse }}}{2}\right)}
$$

\begin{tabular}{|c|c|}
\hline Parameter & Description \\
\hline$P_{t}$ & Transmitted Power \\
$G_{t}$ & Transmitter Gain \\
$\lambda$ & Radar Operating Wavelength \\
$f^{4}$ & Two-way Antenna Pattern \\
$R$ & Slant Range to Clutter (Center) Cell \\
$\sigma_{0}$ & Mean Clutter Reflectivity \\
$\theta_{B}$ & Antenna Pattern Beamwidth \\
$\tau_{p u l s e}$ & Radar Pulse Width $\left(\frac{1}{P R F}\right)$ \\
$C$ & Speed of Light \\
\hline
\end{tabular}

Table A.1: Radar attributes contributing to prediction of $b$, per Eq. (A.1)

Several empirical models have been developed to derive clutter mean reflectivity $\sigma_{0}$. These models provide guidance to a wide range of values likely to be encountered in 
real scenarios, as opposed to offering any precise insight into parametric dependencies. The models also leverage several simplifying assumptions which limits the number of possible situations they can be applied to. Such limitation mainly arises from insufficient knowledge of ocean backscatter at a microwave level [25]. Finally, several empirical prediction models for $\nu$, clutter shape factor, do exists, but all of them are limited to the $\mathrm{X}$-band ( $8 \mathrm{GHz}$ to $10 \mathrm{GHz})$.

In this thesis, we used values for $b$ and $\nu$ based on reported estimations executed on real clutter data, available in [25]. 


\section{Appendix B}

\section{The Hybrid Method to Simulate Ocean Clutter}

In this thesis we simulated ocean clutter using the Hybrid Method developed in [30]. Ocean clutter is statistically modeled by shape parameter $\nu$, scale parameter $b$, spatial and temporal correlation properties.

There are a few constraints on the autocorrelation functions of realizable clutter correlation properties. Let us denote the autocorrelation function as $\rho(\delta)$. The toeplitz matrix of this function, $\boldsymbol{M}$, can be expressed as:

$$
\boldsymbol{M}(i, j) \triangleq \rho(i-j \mid)
$$

for realizable correlation properties, the Toeplitz matrix of $\rho$ must be semi-positive definite. This and other constraints are summarized in Eq. (B.2).

$$
\left\{\begin{array}{l}
\rho(0)=1 \\
\forall \delta:|\rho(\delta)| \leq 1 \\
\boldsymbol{M} \succeq 0
\end{array}\right.
$$

For given values of $\nu$ and $b$, and realizable autocorrelation functions of clutter spatial correlation $\rho_{S}(\cdot)$ and temporal correlation $\rho_{T}(\cdot)$, we can use Hybrid Method to generate spatially correlated clutter data. The syntax of this method, becomes conveniently simple for a subset of $\nu$ values. However, the underlying element in all scenarios is transformation of Gaussian processes to correlated Gamma variables. 


\section{B.1 Shape factor to be an integer}

For integer values of $\nu$, [34] showed the Compound K-distribution can be simplified by a sum of correlated Gamma distributed variables, that is, $\boldsymbol{X} \sim \Gamma(1,1)$. It can be proven [29], that to generate the Gamma variables $x(t)$, with desired correlation $\rho_{S}$, a filter $h(t)$ exists that transforms the white Gaussian noise $u(t)$ to $x(t)$. The PSD of the filter can be obtained using the Fourier synthesis.

$$
\begin{gathered}
u(t) \sim \mathcal{C N}(0,1) \\
S_{h h}(f)=\int_{-\infty}^{\infty} \rho_{S}(\tau) e^{-j 2 \pi f \tau} d \tau \\
H(f)=\sqrt{S_{h h}(f)} \Rightarrow h(t)=\mathcal{F}^{-1}(H(f)) \Rightarrow x(t)=h(t) * u(t)
\end{gathered}
$$

now, for $\nu \in \mathcal{Z}$, we obtain the set of spatially correlated data sequences with:

$$
\begin{gathered}
\forall k=1, \ldots, \nu: \boldsymbol{X}_{k} \sim \Gamma(1,1) \quad \text { Correlated with } \rho_{S} \\
\boldsymbol{Z} \triangleq \sum_{k=1}^{\nu}\left|\boldsymbol{X}_{k}\right|^{2} \Rightarrow \boldsymbol{Z} \sim \Gamma(\nu, 1)
\end{gathered}
$$

for other power factors of the Gamma distribution than unity, we can scale the final $\boldsymbol{Z}$ sequence accordingly.

\section{B.2 Shape factor to be an integer plus 0.5}

The spatially correlated sequence $z[n]$, with shape factor $m \triangleq \nu-0.5$, can be obtained with the method of appendix B.1, since the shape factor would be an integer. In [30] it is proven that the clutter data with the original shape factor $\nu$ is acquired as:

$$
\begin{gathered}
m \triangleq \nu-0.5 \\
\forall k=1, \ldots, m+1: \boldsymbol{X}_{k} \sim \Gamma(1,1) \quad \text { Correlated with } \rho_{S} \\
\boldsymbol{Z} \triangleq\left(\sum_{k=1}^{m}\left|\boldsymbol{X}_{k}\right|^{2}\right)+\Re\left\{\boldsymbol{X}_{m+1}\right\} \Rightarrow \boldsymbol{Z} \sim \Gamma(m+0.5,1)
\end{gathered}
$$

in other words, we generate $\nu+0.5$ sets of correlated Gamma sequences with shape factor 1 , and use the real parts of one of them, to comprise the spatially correlated clutter data. 


\section{B.3 Shape factor to be an arbitrary number}

All values of $\nu$ can be generalized as:

$$
\begin{aligned}
\nu & =\nu_{0}+\Delta \nu \\
\nu_{0} \triangleq m+0.5 \beta \quad & m \in\{0,1,2, \ldots\} ; \beta \in\{0,1\} \\
& 0 \leq \Delta \nu<1
\end{aligned}
$$

Then, a new autocorrelation function, $\eta[k]$, is defined solely for simulation purposes. Whether $\eta[k]$ is a realizable autocorrelation function or not ${ }^{1}$, the method to generate data would differ.

$$
\eta[k] \triangleq \begin{cases}1 & k=0 \\ \rho_{S}[k] \frac{\nu}{\nu_{0}} & k \neq 0\end{cases}
$$

\section{B.3.1 Correlation check to pass}

In this case, the final data sequence can be broken into a correlated Gamma variable $\boldsymbol{Y}$ and an uncorrelated part, $\boldsymbol{\Delta} \boldsymbol{Y}$.

$$
\begin{aligned}
\boldsymbol{Y} & \sim \Gamma\left(\nu_{0}, 1\right) & \text { (correlated with } \eta) \\
\Delta \boldsymbol{Y} & \sim \Gamma(\Delta \nu, 1) & \text { (uncorrelated) } \\
\boldsymbol{Z}=\boldsymbol{Y}+\boldsymbol{\Delta} \boldsymbol{Y} & \sim \Gamma(\nu, 1) & \text { (correlated) }
\end{aligned}
$$

generating uncorrelated Gamma sequences is straightforward, and is already implemented in available software tools. In this thesis, we used the gamrnd function of MATLAB. In previous sections we outlined methods to generate the correlated component, $\boldsymbol{Y}$, too. However, unlike then, the correlation coefficients of $\boldsymbol{Y}$ are given by Eq. (B.14).

\section{B.3.2 Correlation check to fail}

Similar to the previous case, we decompose the final sequence into $\boldsymbol{Y}$ and $\boldsymbol{\Delta} \boldsymbol{Y}$. The difference here is two folds: first, the $\boldsymbol{Y}$ component is correlated with the original coefficients $\rho_{S}$. Second, generation of uncorrelated data sequences is no longer possible with commercial software tools such as MATLAB. To this end, the Memoryless Non-Linear

\footnotetext{
${ }^{1}$ subjected to constraints in Eq. (B.2)
} 
Transform (MNLT) method is utilized, details of which are beyond the scope of this thesis, but available in [30].

The discussion so far has been on generating spatially correlated clutter data sequences. But the Compound K-distribution ensures clutter returns are temporally correlated as well. The implementation of this step is straightforward and trivial, using linear transformation. That is, for $M$ pulses of generated clutter data with length $K$, denoted as $\boldsymbol{z}^{(m)}$, and the given temporal autocorrelation function $\rho_{T}$, we have:

$$
\begin{aligned}
& \forall k \in\{0,1, \ldots, K-1\} \quad \forall m \in\{0,1, \ldots, M-1\} \\
& \boldsymbol{z}^{(m)} \sim \Gamma(\nu, b) \quad \text { Correlated with } \rho_{S} \\
& \boldsymbol{A}_{M \times K}(m, k) \triangleq z^{(m)}[k] \\
& \boldsymbol{M}_{T} \triangleq \operatorname{Toeplitz}\left(\rho_{T}\right) \\
& \tilde{\boldsymbol{A}} \triangleq \boldsymbol{M}_{S}^{1 / 2} \boldsymbol{A} \\
& \tilde{z}^{(m)}[k] \triangleq \tilde{\boldsymbol{A}}(m, k)
\end{aligned}
$$

then, the $\tilde{\boldsymbol{z}}^{(m)}$ sequences are both spatially correlated (using the Hybrid Method) with $\rho_{S}$, and temporally correlated with $\rho_{T}$. Thus, given parameters of a Compound K-distribution, we have established the means to simulate its random variables, suitable for assessing radar performance in presence of ocean clutter. 


\section{Bibliography}

[1] R. Prasad, OFDM for Wireless Communications Systems. Artech House Publishers, 2004.

[2] N. Levanon, "Multifrequency complementary phase-coded radar signal," IEE Proceedings - Radar, Sonar and Navigation, vol. 147, pp. 276-284, Dec 2000.

[3] D. Garmatyuk and M. Brenneman, "Adaptive multicarrier ofdm sar signal processing," IEEE Transactions on Geoscience and Remote Sensing, vol. 49, pp. 3780-3790, Oct 2011.

[4] S. Sen and A. Nehorai, "Target detection in clutter using adaptive ofdm radar," IEEE signal processing letters, vol. 16, pp. 592-595, Jul 2009.

[5] Y. L. Sit, C. Sturm, J. Baier, and T. Zwick, "Direction of arrival estimation using the music algorithm for a mimo ofdm radar," in Radar Conference (RADAR), 2012 IEEE, pp. 0226-0229, IEEE, May 2012.

[6] M. I. Skolnik, "Radar handbook," 1970.

[7] D. Garmatyuk, "Ultrawideband imaging radar based on ofdm: System simulation analysis," in Defense and Security Symposium, pp. 621007-621007, International Society for Optics and Photonics, May 2006.

[8] G. Franken, H. Nikookar, and P. Van Genderen, "Doppler tolerance of ofdm-coded radar signals," in Radar Conference, 2006. EuRAD 2006. 3rd European, pp. 108-111, IEEE, Sep 2006.

[9] Y. L. Sit, C. Sturm, L. Reichardt, T. Zwick, and W. Wiesbeck, "The ofdm joint radar-communication system: An overview," in Proc. Int. Conf. Advances in Satellite and Space Communications (SPACOMM 2011), pp. 69-74, April 2011. 
[10] J. H. Kim, M. Younis, A. Moreira, and W. Wiesbeck, "A novel ofdm chirp waveform scheme for use of multiple transmitters in sar," IEEE Geoscience and Remote Sensing Letters, vol. 10, pp. 568-572, May 2013.

[11] C. Sturm, E. Pancera, T. Zwick, and W. Wiesbeck, "A novel approach to ofdm radar processing," in Radar Conference, 2009 IEEE, pp. 1-4, IEEE, May 2009.

[12] P. Tran, "Person localization in adverse complex environment (place) frequency agility in ofdm active radars," vol. 5, p. 44, Oct 2006.

[13] K. van Caekenberghe, K. F. Brakora, and K. Sarabandi, "A 94 ghz ofdm frequency scanning radar for autonomous landing guidance," in 2007 IEEE Radar Conference, pp. 248-253, April 2007.

[14] J. Fink and F. K. Jondral, "Comparison of ofdm radar and chirp sequence radar," in 2015 16th International Radar Symposium (IRS), pp. 315-320, June 2015.

[15] M. D. Nisar, W. Utschick, H. Nottensteiner, and T. Hindelang, "On channel estimation and equalization of ofdm systems with insufficient cyclic prefix," in 2007 IEEE 65th Vehicular Technology Conference - VTC2007-Spring, pp. 1445-1449, April 2007.

[16] Y. Rahmatallah and S. Mohan, "Peak-to-average power ratio reduction in ofdm systems: A survey and taxonomy," IEEE Communications Surveys Tutorials, vol. 15, pp. 1567-1592, Fourth 2013.

[17] "Federal spectrum use summary," tech. rep., National Telecommunications and Information Administration Office of Spectrum Management, 2010.

[18] A. Oppenheim and R. Schafer, Discrete-time Signal Processing. Always learning, Pearson, 2013.

[19] S. Haykin, An Introduction to Analog and Digital Communications. New York, NY, USA: John Wiley \&amp; Sons, Inc., 1989.

[20] P. Moo, T. Ponsford, D. DiFilippo, R. McKerracher, N. Kashyap, and Y. Allard, "Canada's third generation high frequency surface wave radar system," The Journal of Ocean Technology, vol. 10, pp. 21-28, June 2015.

[21] T. Ponsford and J. Wang, "A review of high frequency surface wave radar for detection and tracking of ships," Turkish Journal of Electrical Engineering 83 Computer Sciences, vol. 18, pp. 409-428, June 2010. 
[22] D. E. Barrick, J. M. Headrick, R. W. Bogle, and D. D. Crombie, "Sea backscatter at hf: Interpretation and utilization of the echo," Proceedings of the IEEE, vol. 62, pp. 673-680, June 1974.

[23] S. Watts, "Radar sea clutter: Recent progress and future challenges," in 2008 International Conference on Radar, pp. 10-16, Sept 2008.

[24] S. Watts, "Specification and measurement of performance for airborne maritime surveillance radars," in Radar 99. International conference, May 1999.

[25] I. Antipov, "Simulation of sea clutter returns," tech. rep., DTIC Document, 1998.

[26] C. J. Baker, "K-distributed coherent sea clutter," IEE Proceedings F - Radar and Signal Processing, vol. 138, pp. 89-92, April 1991.

[27] L. Devroye, "Sample-based non-uniform random variate generation," in Proceedings of the 18th conference on Winter simulation, pp. 260-265, ACM, Dec 1986.

[28] S. Watts and K. Ward, "Spatial correlation in k-distributed sea clutter," in IEE Proceedings F (Communications, Radar and Signal Processing), vol. 134, pp. 526-532, IET, Oct 1987.

[29] K. D. Ward, S. Watts, and R. J. Tough, Sea clutter: scattering, the K distribution and radar performance, vol. 20. IET, 2006.

[30] Y. Dong, L. Rosenberg, and G. V. Weinberg, "Generating correlated gamma sequences for sea-clutter simulation," tech. rep., DTIC Document, Mar 2012.

[31] I. R. Joughin, D. B. Percival, and D. P. Winebrenner, "Maximum likelihood estimation of $\mathrm{k}$ distribution parameters for sar data," IEEE transactions on Geoscience and Remote Sensing, vol. 31, pp. 989-999, Sep 1993.

[32] M. Horst, F. Dyer, and M. Tuley, "Radar sea clutter model," in Antennas and Propagation, vol. 1, pp. 6-10, 1978.

[33] D. W. K. Ng, E. S. Lo, and R. Schober, "Wireless information and power transfer: Energy efficiency optimization in ofdma systems," IEEE Transactions on Wireless Communications, vol. 12, pp. 6352-6370, Dec 2013.

[34] B. C. Armstrong and H. D. Griffiths, "Modelling spatially correlated k-distributed clutter," Electronics Letters, vol. 27, pp. 1355-1356, July 1991. 